\title{
SOLUTION OF CONTAMINANT TRANSPORT WITH ADSORPTION IN POROUS MEDIA BY THE METHOD OF CHARACTERISTICS
}

\author{
JOZEF KACUR ${ }^{1}$ AND Roger VAN KeER ${ }^{2}$
}

\begin{abstract}
A new approximation scheme is presented for the mathematical model of convectiondiffusion and adsorption. The method is based on the relaxation method and the method of characteristics. We prove the convergence of the method and present some numerical experiments in 1D. The results can be applied to the model of contaminant transport in porous media with multi-site, equilibrium and non-equilibrium type of adsorption.
\end{abstract}

Mathematics Subject Classification. 65M25, 65M12.

Received: May 30, 2000. Revised: June 6, 2001.

\section{INTRODUCTION}

We consider the mathematical model for solute transport in porous media, introduced in [21]. This is governed by the differential equations

$$
\begin{array}{rl}
\theta \partial_{t} u+\varrho_{1} \partial_{t} \Psi(u)+\varrho_{2} \int_{\Lambda} \partial_{t} v & \mathrm{~d} \lambda+\bar{q} \cdot \nabla u-\operatorname{div}(D(t, x) \nabla u)=G \\
& \text { in } \quad(t, x) \in I \times \Omega \equiv Q_{T}, I=(0, T), T<\infty,
\end{array}
$$

and

$$
\partial_{t} v=f(\lambda, u, v) \quad \text { in } \Lambda \times Q_{T}
$$

along with the boundary conditions

$$
\begin{array}{rll}
(\bar{q} u-D(t, x) \nabla u) \cdot \bar{\nu} & =g & \text { on } I \times \partial \Omega_{1}, \\
\nabla u . \bar{\nu} & =0 & \text { on } I \times \partial \Omega_{2},
\end{array}
$$

$(\bar{\nu}$ is the unite outward normal vector to $\partial \Omega)$ and along with the initial conditions

$$
u=u_{0} \quad \text { on }\{0\} \times \Omega, \quad v=v_{0} \quad \text { on }\{0\} \times(\Lambda \times \Omega) .
$$

Keywords and phrases. Relaxation method, method of characteristics, contaminant transport, convection-diffusion with adsorption.

${ }^{1}$ Faculty of Mathematics and Physics, Comenius University Mlynska Dolina, 84815 Bratislava, Slovakia.

e-mail: kacur@fmph.uniba.sk

2 Ghent University, Department of Mathematical Analysis, Galglaan 2, 9000 Gent, Belgium.

e-mail: roger.vankeer@cage.rug.ac.be 
Here $\partial \Omega_{1}, \partial \Omega_{2}$ are open, non-overlapping subsets of the Lipschitz continuous boundary $\partial \Omega$ of the bounded domain $\Omega \subset \mathbb{R}^{N}$, with $\operatorname{mes}_{N-1} \partial \Omega_{1}+\operatorname{mes}_{N-1} \partial \Omega_{2}=\operatorname{mes}_{N-1} \partial \Omega$.

The contaminant dissoluted in the groundwater with concentration $u$ is transported and adsorbed by the porous media skeleton. A multiple site adsorption is considered. The mass of the porous media is subdivided into two parts related to adsorption sites where the reaction is realized in equilibrium and nonequilibrium modes, respectively. The equilibrium reaction is represented by $\Psi(u)$ with a so called adsorption isotherm $\Psi$. The adsorbed contaminant concentration due to the nonequilibrium reaction is represented by $v=v(\lambda, x, t)$ for $\lambda \in \Lambda$, where $\Lambda$ characterizes chemically qualitatively different adsorption sites at the skeleton surfaces related to a unit volume (representative control volume-REV) at the point $x$. The kinetics of the nonequilibrium adsorption is governed by (2) where $f$ is nonincreasing in $v$ (at fixed $\lambda, u$ ).

We assume $(\Lambda, \mu)$ to be a measure space with $\mu \geq 0$ and $\mu(\Lambda)=1$ see [13]. Equation (1) represents the conservation of the total mass of the contaminant. Here, it is implicitly assumed that the conservation of liquid mass takes place:

$$
\partial_{t} \theta+\operatorname{div} \bar{q}=0 \text { in } I \times \Omega, \quad \bar{q} \cdot \bar{\nu}=h \quad \text { on } I \times \partial \Omega
$$

for the volumetric water content $\theta$ and flux $\bar{q}$.

The adsorption term in (1) reflects the heterogenity of the adsorbent surface grains (rescaled grain surface, e.g. REV) and is a generalization of the adsorption process considered in [11]. There, the REV is subdivided into $m$ chemically different collections of adsorption sites, where $\lambda_{i}(i \in\{1, \cdots, m\})$ represents their relative size in the REV and $v_{i}=v\left(\lambda_{i}, x, t\right)$ represents the adsorbed concentration of the $i$-th site. Then, $\sum_{i=1}^{m} \lambda_{i}=1$ and $v=\sum_{i=1}^{m} v_{i} \lambda_{i}$, which is a discrete form of the adsorption model in (1),(2).

In transport models in porous media $D$ takes the form

$$
D_{i j}=\left\{D_{\mathrm{mol}}+\alpha_{T}|\bar{q}|\right\} \delta_{i j}+\left(\alpha_{L}-\alpha_{T}\right) \frac{q_{i} q_{j}}{|\bar{q}|}, i, j=1, \ldots, N
$$

where $D_{\text {mol }}$ is the molecular diffusivity of the contaminant in the fluid and $\alpha_{T}, \alpha_{L}$ are the transversal and longitudinal lengths, respectively - see [5]. Finally, the bulk densities $\rho_{1}, \rho_{2}$ correspond to the different kinds of adsorption sites.

In [21] the uniqueness of the variational solution of (1-4) has been proved under rather general assumptions:

$$
\begin{gathered}
D \equiv D(x) ; 0 \leq \rho_{1}, \rho_{2} \in L_{\infty}(\Omega) ; G \in L_{1}\left(Q_{T}\right) ; g \in L_{1}\left(I \times \partial \Omega_{1}\right) \\
h \in L_{1}(I \times \partial \Omega) ; 0 \leq \theta \in L_{\infty}\left(Q_{T}\right) ; \bar{q} \in\left[L_{2}\left(Q_{T}\right)\right]^{N} ; f\left(\lambda, x, u_{1}, v_{1}\right) \geq \\
f\left(\lambda, x, u_{2}, v_{2}\right) \text { for } u_{1} \geq u_{2}, v_{1} \leq v_{2}, \lambda \in \Lambda, x \in \Omega ; h \geq 0 \text { on } I \times \partial \Omega_{2} ;
\end{gathered}
$$

one of the following conditions is satisfied : $\Psi$ is strictly increasing and either

$$
\rho_{1}>0 \text { or } \theta>0 \text { or } \operatorname{mes}_{N-1} \partial \Omega_{2}>0 ;(5) \text { is satisfied in a weak sense. }
$$

A typical example of chemical kinetics is

$$
f(\lambda, x, u, v):=k(\lambda, x)(\varphi(\lambda, u)-v)
$$

with a rate parameter $k$ and adsorption isotherm $\varphi(\lambda, u)$. The function $\varphi$ has similar properties as the sorption isotherm $\Psi$. The equilibrium sorption process corresponds to (6) for $k \rightarrow \infty$, i.e., $v=\psi(u)$. The most common sorption isotherms are of Langmuir type: $\psi(u)=\frac{k_{1} u}{1+k_{2} u}$, or Freundlich type: $\Psi(u)=k_{3} u^{p}$, with $0<p$. If $\Psi$ is of Freundlich type with $0<p<1$, then the parabolic term in (1) degenerates since $\Psi^{\prime}(0)=\infty$ and in this case the solutions of (1) exhibit similar properties as the solutions of the "porous medium equation". In that case the solution possesses a limited regularity at the boundary of its support (i.e., the interface). The interface 
propagates with finite speed. Moreover, the presence of the convective term (eventually dominant) gives rise to sharp fronts at the interface. This makes the numerical treatment very difficult.

There are many papers discussing the existence, uniqueness, regularity, asymptotic behaviour and numerical approximation of contaminant transport problems in porous media, which are special cases of $(1-4),(\bar{q}$ and $\theta$ are Lipschitz continuous, $v$ is independent of $\lambda$, adsorption kinetics (6) is considered only, $\rho_{1}$ or $\rho_{2}$ vanishes throughout $\Omega$ ). See $[2-4,7-10,13,15,19,26]$, among others.

The main goal of this paper is to suggest an efficient numerical approximation based on the method of relaxation (to control the degenerated parabolic term) and on the method of characteristics (to control the eventually dominant convective term). We will prove the convergence of the suggested approximation scheme for $(1-4)$.

For the relaxation techniques we follow [15-17]. The method of characteristics has been initiated by [26], [12] and is then intensively developed in the last two decades. Also in contaminant transport problems the method of characteristics has been applied in [11] and [4], where $\varrho_{1} \equiv 0$, and in [9], where $\varrho_{2} \equiv 0$. There, the Lipschitz continuity of $\theta$ and $\bar{q}$ has been assumed. Here, we assume only the boundedness of $\theta$ and $\bar{q}$ and the Lipschitz continuity of $\theta$ in the $t$-variable. The velocity field (discharge) $\bar{q}$ and the volumetric water content $\theta$ are usually solutions of an underground water flow problem (modelled by the strongly nonlinear Richard's equation) and are not very smooth in $x$.

Our concept of approximation of (1-4) is as follows. We use a nonstandard time discretization where $u_{i} \approx$ $u\left(t_{i}, x\right), v_{i} \approx v\left(t_{i}, x\right)$ with $t_{i}=i \tau, \tau=\frac{T}{n}$ (time step), will be determined from the elliptic equation (7) below. The method of characteristics will be implemented by approximated characteristics $\varphi_{i}(x)=x-\tau \omega_{h} * \frac{\bar{q}_{i}}{\theta_{i}}$ on the time interval $t \in\left(t_{i-1}, t_{i}\right)$, where $\bar{q}_{i}=\bar{q}\left(t_{i}, x\right), \theta_{i}=\theta\left(t_{i}, x\right)$ and where $\omega_{h}$ is a mollifier, e.g., $\omega_{h}(x)=\frac{1}{h^{N}} \omega_{1}\left(\frac{x}{h}\right)$, with

$$
\omega_{1}(x)=\frac{1}{\kappa}\left\{\begin{array}{ll}
\exp \left(\frac{|x|^{2}}{|x|^{2}-1}\right) & |x| \leq 1, \\
0, & |x| \geq 1
\end{array}, \quad \int_{R^{N}} \omega_{1}(x) \mathrm{d} x=1\right.
$$

and

$$
g * z(x)=\int_{R^{N}} g(x-\xi) z(\xi) \mathrm{d} \xi
$$

We will take $h=\tau^{\omega}$ with a fixed parameter $\omega \in(0,1)$. At the time level $t=t_{i}$ we approximate the transport part of the convection-diffusion-adsorption process by $u_{i-1} \circ \varphi^{i}(x) \equiv u_{i-1}\left(\varphi^{i}(x)\right)$, which represents the shifting of the profile $u_{i-1}$ along the approximated characteristics $\varphi^{i}$.

We determine $u_{i}$ from elliptic problems (containing diffusion and adsorption) in one of the following schemes.

\section{Scheme I.}

$$
\begin{gathered}
\theta_{i} \frac{u_{i}-u_{i-1} \circ \varphi_{i}}{\tau}+\varrho_{1} \mu_{i} \frac{u_{i}-u_{i-1}}{\tau}+\varrho_{2} \int_{\Lambda} f\left(\lambda, u_{i}, v_{i-1}\right) \mathrm{d} \lambda-\operatorname{div}\left(D_{i} \nabla u_{i}\right)=G_{i} \quad \text { in } \Omega \\
\int_{\Lambda} \frac{v_{i}-v_{i-1}}{\tau} \mathrm{d} \lambda=\int_{\Lambda} f\left(\lambda, u_{i}, v_{i-1}\right) \mathrm{d} \lambda, \quad \text { a.e. } x \in Q_{T},
\end{gathered}
$$

along with the boundary conditions

$$
\begin{aligned}
\left(\bar{q}_{i} u_{i}-D_{i} u_{i}\right) \cdot \bar{\nu} & =g_{i} \quad \text { on } \partial \Omega_{1} \\
\nabla u_{i} \cdot \bar{\nu} & =0 \text { on } \partial \Omega_{2} .
\end{aligned}
$$

Here, $0 \leq \mu_{i} \in L_{\infty}(\Omega)$ is a relaxation function which has to satisfy the "convergence" condition

$$
\left\|\mu_{i}-\frac{\Psi_{n}\left(u_{i}\right)-\Psi_{n}\left(u_{i-1}\right)}{u_{i}-u_{i-1}}\right\|_{\infty} \leq \tau,
$$


$\|\cdot\|_{\infty}$ being the $L_{\infty}$-norm and $\Psi_{n}$ being a regularization of $\Psi,\left(\Psi_{n} \rightarrow \Psi\right.$ for $\left.n \rightarrow \infty\right)$, which we will specify in the assumption $H_{2}$ ) (Sect. 2).

\section{Scheme II.}

$$
\begin{gathered}
\theta_{i} \frac{u_{i}-u_{i-1} \circ \varphi_{i}}{\tau}+\varrho_{1} \mu_{i} \frac{u_{i}-u_{i-1}}{\tau}+\varrho_{2} \int_{\Lambda} f\left(\lambda, u_{i-1}, v_{i-1}\right) \mathrm{d} \lambda-\operatorname{div}\left(D_{i} \nabla u_{i}\right)=G_{i} \quad \text { in } \Omega, \\
\int_{\Lambda} \frac{v_{i}-v_{i-1}}{\tau} \mathrm{d} \lambda=\int_{\Lambda} f\left(\lambda, u_{i-1}, v_{i-1}\right) \mathrm{d} \lambda, \quad \text { a.e. } x \in Q_{T},
\end{gathered}
$$

along with the boundary conditions (8) and "convergence condition" (9). This scheme is explicit with respect to the adsorption term, in contrast to Scheme I.

Approximation Schemes I and II are implicit, since $u_{i}, \mu_{i}$ are coupled in $(7,9)$ and $(9,10)$, respectively. We can modify Schemes I and II into an explicit form by means of an iteration-relaxation process with parameter $k=1, \ldots$

\section{Scheme $\mathbf{I}_{k}$.}

$$
\theta_{i} \frac{u_{i, k}-u_{i-1} \circ \varphi_{i}}{\tau}+\varrho_{1} \mu_{i, k-1} \frac{u_{i, k}-u_{i-1}}{\tau}+\varrho_{2} \int_{\Lambda} f\left(\lambda, u_{i, k-1}, v_{i-1}\right) \mathrm{d} \lambda-\operatorname{div}\left(D_{i} \nabla u_{i, k}\right)=G_{i},
$$

along with the boundary conditions (8) for $u_{i, k}$. Then, we put

$$
\mu_{i, k}:=\frac{\Psi_{n}\left(u_{i, k}\right)-\Psi_{n}\left(u_{i-1}\right)}{u_{i, k}-u_{i-1}}, \quad \mu_{i, 0}=\Psi_{n}^{\prime}\left(u_{i-1}\right), \quad k=1, \ldots .
$$

If $\left\|\mu_{i, k_{0}}-\mu_{i, k_{0}-1}\right\|_{\infty}<\tau$, then we take $\mu_{i}:=\mu_{i, k_{0}-1}$ and $u_{i}:=u_{i, k_{0}}$.

A simple iteration process in (10) can be taken as follows:

Scheme $\mathbf{I I}_{k}$.

$$
\theta_{i} \frac{u_{i, k}-u_{i-1} \circ \varphi_{i}}{\tau}+\varrho_{1} \mu_{i, k-1} \frac{u_{i, k}-u_{i-1}}{\tau}+\varrho_{2} \int_{\Lambda} f\left(\lambda, u_{i-1}, v_{i-1}\right) \mathrm{d} \lambda-\operatorname{div}\left(D_{i} \nabla u_{i, k}\right)=G_{i} \quad \text { in } \Omega,
$$

along with the BC's (8) and along with (12).

Remark 1.1. Another, however numerically more costly, linearization can be considered when in (11) we replace $f\left(\lambda, u_{i, k-1}, v_{i-1}\right)$ by $f\left(\lambda, u_{i, k-1}, v_{i-1}\right)+f_{u}^{\prime}\left(\lambda, u_{i, k-1}, v_{i-1}\right)\left(u_{i, k}-u_{i, k-1}\right)$. The adsorption term can also be included take in the iteration process, when replacing $f\left(\lambda, u_{i, k-1}, v_{i-1}\right)$ by $f\left(\lambda, u_{i, k-1}, v_{i, k-1}\right)$ and $f_{u}^{\prime}\left(\lambda, u_{i, k-1}, v_{i-1}\right)$ by $f_{u}^{\prime}\left(\lambda, u_{i, k-1}, v_{i, k-1}\right)$, and when next defining $v_{i, k}$ by:

$$
\int_{\Lambda} \frac{v_{i, k}-v_{i-1}}{\tau} \mathrm{d} \lambda=\int_{\Lambda} f\left(\lambda, u_{i, k-1}, v_{i, k-1}\right) \mathrm{d} \lambda, k=1, \ldots
$$

This would correspond to an implicit scheme, where in (7) the term $f\left(\lambda, u_{i}, v_{i-1}\right)$ is replaced by $f\left(\lambda, u_{i}, v_{i}\right)$. This type of approximation requires the Lipschitz continuity of $f$.

The approximations in Scheme $\mathrm{I}_{k}$ and $\mathrm{Scheme} \mathrm{II}_{k}$, respectively, reduce the original problem to solving a sequence of linear elliptic problems.

In Section 2 we introduce the underlying assumptions and the variational formulation of (1-4) and of (7-8). In Section 3 we discuss the convergence of the iterations in Scheme $\mathrm{II}_{k}$ with respect to $k$. The convergence of the approximation Schemes I and II is proved in Section 4. In Section 5 some generalizations concerning the structure of $f$ are considered. We shortly discuss the full discretization scheme of (1-4) in Section 6. Some simple numerical experiments are presented in Section 7. 


\section{Assumptions AND VARIATIONAL FORMUlations}

In the sequel, c denotes a generic positive constant, $V$ denotes the Sobolev space $W_{2}^{1}(\Omega)$ and $V^{*}$ its dual space with duality $\langle w, u\rangle$ for $w \in V^{*}, u \in V$. We denote $(u, v):=\int_{\Omega} u v \mathrm{~d} x$ for $u, v \in L_{2} \equiv L_{2}(\Omega),(u, v)_{\Gamma}:=\int_{\Gamma} u v \mathrm{~d} x$ for $u, v \in L_{2}(\partial \Omega), \quad(\Gamma \subset \partial \Omega)$. By $L_{2}(I, V), L_{2}\left(I, L_{2}\right)$ we denote functional spaces of abstract functions $u: I \rightarrow$ $V$, resp. $u: I \rightarrow L_{2}$ - see [22]. We denote by $\|\cdot\|_{0},\|\cdot\|,\|\cdot\|_{*}$ and $\|\cdot\|_{\Gamma}$ the norms in the spaces $L_{2}, V, V^{*}$ and $L_{2}(\Gamma)$, respectively. Finally, we denote

$$
b(t, u):=\theta(t, x) u+\varrho_{1}(x) \Psi(u) .
$$

Definition 2.1. $u \in L_{2}(I, V), v \in L_{2}\left(I, L_{2}(\Lambda \times \Omega)\right)$ is a variational solution of (1-4) if

i) $b(t, u) \in L_{1}\left(I, L_{1}(\Omega)\right), \partial_{t} b \in L_{2}\left(I, V^{*}\right), \partial_{t} v \in L_{2}\left(I, L_{2}(\Lambda \times \Omega)\right)$,

ii)

$$
\begin{gathered}
\int_{I}\left\langle\partial_{t} b(t, u), w\right\rangle \mathrm{d} t-\int_{I}\left(\partial_{t} \theta u, w\right) \mathrm{d} t+\int_{I} \int_{\Lambda}(f(\lambda, u, v), w) \mathrm{d} \lambda \mathrm{d} t \\
+\int_{I}(\bar{q} . \nabla u, w) \mathrm{d} t+\int_{I}(D \nabla u, \nabla w) \mathrm{d} t-\int_{I}(h, w)_{\partial \Omega_{1}} \mathrm{~d} t+\int_{I}(g, w)_{\partial \Omega_{1}} \mathrm{~d} t \\
=\int_{I}(G, w) \mathrm{d} t \quad \forall w \in L_{2}(I, V)
\end{gathered}
$$

and

$$
\int_{\Lambda}\left(\partial_{t} v, \xi\right) \mathrm{d} \lambda=\int_{\Lambda}(f(\lambda, u, v), \xi) \mathrm{d} \lambda \quad \forall \xi \in L_{2}\left(I, L_{2}(\Lambda \times \Omega)\right)
$$

iii)

$$
\left.\int_{I}\left\langle\partial_{t} b(t, u), \xi\right)\right\rangle \mathrm{d} t=-\int_{I} \int_{\Omega}\left(b(t, u)-b\left(0, u_{0}\right)\right) \partial_{t} \xi \mathrm{d} x \mathrm{~d} t \quad \forall \xi \in L_{2}(I, V),
$$

with

$$
\partial_{t} \xi \in L_{\infty}\left(Q_{T}\right) \text { and } \xi(x, T)=0
$$

In Definition 2.1, equation (5) is implicitly assumed to take place. This is a modification of the variational solution in [21], which allows us to assume less regularity for $\theta$ and $q$ in (5). In our notation the identity $i i)$ is considered in the form

$$
\begin{gathered}
\int_{I}\left\langle\partial_{t} b(t, u), w\right\rangle \mathrm{d} t+\int_{I} \int_{\Lambda}(f(\lambda, u, v), w) \mathrm{d} \lambda \mathrm{d} t-\int_{I}(\bar{q} u-D \nabla u, \nabla w) \mathrm{d} t \\
+\int_{I}(g, w)_{\partial \Omega_{1}} \mathrm{~d} t+\int_{I}(h, w)_{\partial \Omega_{2}} \mathrm{~d} t=\int_{I}(G, w) \mathrm{d} t
\end{gathered}
$$

which under (5) (with $\partial_{t} \theta \in L_{\infty}\left(Q_{T}\right), \operatorname{div} \bar{q} \in L_{2}\left(Q_{T}\right)$ ) is equivalent with $\left.i i\right)$. The uniqueness result in [21] for the variational solution is very important for the numerical analysis and we shall use it. The variational formulation in Definition 1 is suitable for our concept of approximation applying the method of characteristics.

The solution in (7) is also understood in the variational form :

$$
\begin{gathered}
\left(\theta_{i} \frac{u_{i}-u_{i-1} \circ \varphi_{i}}{\tau}, w\right)+\left(\varrho_{1} \mu_{i} \frac{u_{i}-u_{i-1}}{\tau}, w\right)+\int_{\Lambda}\left(\varrho_{2} f\left(\lambda, u_{i}, v_{i-1}\right), w\right) \mathrm{d} \lambda \\
-(h, w)_{\partial \Omega_{1}}+\left(g_{i}, w\right)_{\partial \Omega_{1}}+\left(D_{i} \nabla u_{i}, \nabla w\right) \stackrel{=}{=}\left(G_{i}, w\right), \quad \forall w \in V
\end{gathered}
$$

provided that $(7)_{2}$ holds for a.e. $x \in Q_{T}$. When using an explicit modification in the reaction term, we take

$$
\begin{gathered}
\left(\theta_{i} \frac{u_{i}-u_{i-1} \circ \varphi_{i}}{\tau}, w\right)+\left(\varrho_{1} \mu_{i} \frac{u_{i}-u_{i-1}}{\tau}, w\right)+\int_{\Lambda}\left(\varrho_{2} f\left(\lambda, u_{i-1}, v_{i-1}\right), w\right) \mathrm{d} \lambda \\
-(h, w)_{\partial \Omega_{1}}+\left(g_{i}, w\right)_{\partial \Omega_{1}}+\left(D_{i} \nabla u_{i}, \nabla w\right)=\left(G_{i}, w\right), \quad \forall w \in V
\end{gathered}
$$


and in $(7)_{2}$ we replace $u_{i}$ by $u_{i-1}$. For $\bar{q}_{i}, \theta_{i}, g_{i}, h_{i}$ we can take the corresponding $L_{2}$-average, e.g., $\bar{q}_{i}=$ $\frac{1}{\tau} \int_{t_{i-1}}^{t_{i}} \bar{q}(s) \mathrm{d} s$ and similarly for $\theta_{i}, g_{i}, h_{i}$.

Assumptions. To guarantee the convergence of the approximation scheme (7-9) and of the scheme (8-10) we shall assume

$\left.\mathrm{H}_{1}\right) \theta(t, x) \geq \theta^{\circ}>0$ is continuous in $I \times \Omega$ and Lipschitz continuous in $t \in I$, i.e., $\left|\theta(t, x)-\theta\left(t^{\prime}, x\right)\right| \leq$ $c\left|t-t^{\prime}\right| ; \bar{q} \in L_{\infty}\left(Q_{T}\right) ; h \in L_{\infty}(I \times \partial \Omega)$.

$\left.\mathrm{H}_{2}\right)$ D is continuous on $\bar{Q}_{T}$ and satisfies

$$
(D \xi, \xi) \geq c_{e}|\xi|^{2} \quad \forall \xi \in R^{N} .
$$

$\left.\mathrm{H}_{3}\right) \mathrm{G}$ is continuous on $\overline{Q_{T}} ; \mathrm{g}$ is continuous on $\overline{I \times \partial \Omega_{1}} ; \varrho_{1}, \varrho_{2} \in L_{\infty}(\Omega)$ and $\varrho_{1}, \varrho_{2} \geq 0$.

$\left.\mathrm{H}_{4}\right) f(\lambda, x, u, v)$ is continuous and bounded on $\Lambda \times R \times R$ and

$$
-K \leq f_{v}^{\prime}(\lambda, x, u, v) \leq 0 \quad \text { for } \quad(\lambda, x, s, p) \in \Lambda \times \Omega \times R \times R .
$$

$\left.\mathrm{H}_{5}\right) \Psi$ is nondecreasing $(\Psi(0)=0)$ and there exists $\Psi_{n}(s) \in C^{1}(R)$ such that $\Psi_{n}(s) \rightarrow \Psi(s)$ locally uniformly for $s$ in a bounded set and such that

$$
0 \leq \Psi_{n}^{\prime}(s) \leq c \tau^{-\alpha} ; \Psi_{n}(0)=0 ; \sup _{n,|s| \leq K}\left|\Psi_{n}(s)\right| \leq C(K)<\infty .
$$

$\left.\mathrm{H}_{6}\right) u_{0} \in L_{2}(\Omega), v_{0} \in L_{2}(\Lambda)$.

By $\varphi_{i},\left(\varphi_{i}(x)=x-\tau \omega_{h} * \frac{\bar{q}_{i}}{\theta_{i}}\right)$, we map $\Omega$ into $\Omega_{i} \subset \Omega^{\star}$, where $\Omega^{\star} \supset \bar{\Omega}$ is a small neighborhood of $\Omega$, provided $\tau \leq \tau_{0}$. To apply the method of characteristics it is crucial that $\varphi_{i}$ and its inverse $\varphi_{i}^{-1}$ are Lipschitz continuous uniformly for $n,(i=1, \ldots, n)$. If $\varphi_{i}(x)$ does not belong to $\Omega$, then we extend $u_{i-1} \in V$ into $\tilde{u}_{i-1} \in W_{2}^{1}\left(\Omega^{*}\right)$ and we replace $u_{i-1} \circ \varphi_{i}$ by $\tilde{u}_{i-1} \circ \varphi_{i}$. The prolongation of $u_{i-1}$ can be realized in such a way that

$$
\left\|u_{i-1}\right\|_{W_{2}^{1}\left(\Omega^{*}\right)} \leq c\left\|u_{i-1}\right\|_{W_{2}^{1}(\Omega)} \quad\left(c \text { is independent on } u_{i-1}\right)
$$

- see [24] (prolongation of Nikolskij).

Lemma 2.2. If $\omega \in(0,1)$ then $\varphi_{i}$ is one to one and it holds that

$$
\frac{1}{2}|x-y| \leq\left|\varphi_{i}(x)-\varphi_{i}(y)\right| \leq 2|x-y|, \quad i=1, \ldots, n,
$$

uniformly for $x, y \in \Omega$, provided that $\tau \leq \tau_{0}$.

Proof. Since $\varphi_{i}(x)=x-\omega_{h} * \frac{\bar{q}_{i}}{\theta_{i}}$ and $\left\|\frac{\bar{q}_{i}}{\theta_{i}}\right\|_{\infty} \leq M<\infty$, for $i=1, \ldots, n$, we have

$$
\left\|\omega_{h} * \frac{\bar{q}_{i}}{\theta_{i}}\right\|_{\infty} \leq M \quad \text { and } \quad\left\|\nabla_{x} \omega_{h} * \frac{\bar{q}_{i}}{\theta_{i}}\right\|_{\infty} \leq \frac{c}{h}\left\|\frac{\bar{q}_{i}}{\theta_{i}}\right\|_{\infty} \leq \frac{c M}{h} .
$$

Since $h=\tau^{\omega}$, we have

$$
\left(1-\tau^{1-\omega} c M\right)|x-y| \leq \varphi_{i}(x)-\varphi_{i}(y)\left|\leq\left(1+\tau^{1-\omega} c M\right)\right| x-y \mid, \quad \forall x, y \in \Omega,
$$

which implies our assertion.

The solution of (11) and (13), respectively, is understood in the variational sense. The existence and uniqueness of the variational solution $u_{i, k} \in V$ of (13) is guaranteed by a Lax-Millgram argument. If, additionally to 
$\left.H_{4}\right), f(\lambda, u, v)$ is nondecreasing in $u$ then there exists a unique variational solution of (11) too, because of the theory of monotone operators.

Under the "optimal" variational solution of (7-9) or of (8-10) we understand $u_{i} \in V$ such that $\mu_{i}=$ $\frac{\Psi_{n}\left(u_{i}\right)-\Psi_{n}\left(u_{i-1}\right)}{u_{i}-u_{i-1}}$. The existence of a unique (optimal) variational solution of (8-10) is again guaranteed by the theory of monotone operators, since we replace $\mu_{i} \frac{u_{i}-u_{i-1}}{\tau}$ by $\frac{\Psi_{n}\left(u_{i}\right)-\Psi_{n}\left(u_{i-1}\right)}{\tau}$ with monotone $\Psi_{n}$. If $f$ is nondecreasing in $u$, then there exists a unique "optimal" variational solution of $(7-9)$.

The convergence of (12-13) to an "optimal" variational solution of (9-10) is discussed, under additional structural and regularity assumptions, in the next section.

\section{Convergence of Scheme $\mathrm{II}_{k}$}

Let us denote by $P_{i}$, the Dirichlet boundary value problems $(9,10)$ with $u_{i}=w$ on $\partial \Omega$. Likewise, $P_{i, k}$ is the Dirichlet problem $(12,13)$ with $u_{i, k}=w$ on $\partial \Omega$. We consider the following restrictions:

(i) $w$ is Lipschitz continuous on $\partial \Omega$ and there exists $u_{w} \in W_{2}^{1}(\Omega) \cap C^{0, \frac{1}{2}}(\bar{\Omega}) \quad\left(C^{0, \beta}\right.$ is the set of all $\beta$ - Holder continuous functions) such that $\left.u_{w}\right|_{\partial \Omega} \equiv w$;

(ii) $D_{i} \equiv D(x)$ is symmetric:

(iii) $\mid \Psi_{n}^{\prime \prime}(s) \| \leq c \tau^{-\gamma}, \gamma \in(0,1)$.

Following [17] we can prove

Lemma 3.1. Let $\left.\left.H_{1}\right)-H_{5}\right)$ and $(i--i i i)$ be satisfied. We assume that the variational solutions $u_{i}$ of $P_{i}$ are uniformly bounded in $C^{0, \alpha}(\bar{\Omega})$ for some $\alpha \in(0,1)$. If $\frac{\alpha}{2}-\gamma>0$, then there exists $\tau_{0}>0$ such that $u_{i, k} \rightarrow u_{i}$ in $C(\bar{\Omega})$ for $k \rightarrow \infty$, where $u_{i}$ is an "optimal" variational solution of $P_{i}$ (i.e, $\mu_{i}=\frac{\Psi_{n}\left(u_{i}\right)-\Psi_{n}\left(u_{i-1}\right)}{u_{i}-u_{i}-1}$ is satisfied). There exists $k_{0}<\infty$ such that $u_{i}:=u_{i, k_{0}}, \mu_{i}:=u_{i, k_{0}-1}$ satisfies $P_{i}$.

Proof. We put $\lambda(v ; z):=\theta+\varrho_{1} \mu(v ; z) \equiv \theta+\varrho_{1} \frac{\Psi_{n}(v)-\Psi_{n}(z)}{v-z}$ and $F:=\theta \frac{z-z \circ \varphi}{\tau}$. We rewrite (10) in the form

$$
\lambda\left(v ; u_{i-1}\right)\left(u-u_{i-1}\right)-\tau \nabla(D \nabla u)=\tau\left(G_{i}+F_{i}\right),
$$

where

$$
F_{i}=\theta_{i} \frac{u_{i-1}-u_{i-1} \circ \varphi_{i}}{\tau}, \quad v=u_{i, k-1}, \quad u=u_{i, k}
$$

which is of the form (5.1) in [17]. An "optimal" solution of $P_{i}$ is the solution of (16) with $v=u$. The results in [17] (Lem. 5.2 and Th. 5.1) can be adapted in our case if the inequality $\tau\left\|f_{i}\right\|_{\infty} \leq c \tau^{\alpha / 2}$ takes place (see the proof of Lem. 5.2 in [17]). The assumption $u_{i-1} \in C^{0, \alpha}$ and hypothesis $H_{1}$ ) lead to the estimate

$$
\tau\left\|F_{i}\right\|_{\infty} \leq\left\|u_{i-1}\right\|_{C^{0, \alpha}} \tau^{\alpha / 2}\left\|\omega_{h} * \frac{\bar{q}_{i}}{\theta_{i}}\right\|_{\infty} \leq c \tau^{\alpha / 2}
$$

In our case the Lipschitz constant $L_{\lambda}$ of $\lambda(v ; z)$ with respect to $v$ is estimated by $L_{\lambda} \leq c \tau^{-\gamma}$ which follows from (iii). Moreover, in our case $\lambda$ is regular from below, i.e. $\lambda(v ; z) \geq \theta_{0} \geq 0$ and the corresponding parameter $d$ in [17] equals zero. Then, the result follows from Theorem 5.1 in [17].

Now we prove that under the assumptions of Lemma 3.1 , the estimate $\left\|\nabla u_{i}\right\|_{0} \leq c, \forall n,(i=1, \ldots, n)$, holds from which it follows that $\left\|u_{i}\right\|_{C^{0, \alpha}(\bar{\Omega})} \leq c$ in the 1D-case. For simplicity we denote

$$
\delta u_{i}:=\frac{u_{i}-u_{i-1}}{\tau}
$$

Lemma 3.2. Retain the assumptions $\left.\left.H_{1}\right)-H_{5}\right)$ and $(i-i i i)$. Moreover, assume that $u_{0} \in W_{2}^{1}, \partial_{t} h \in$ $L_{2}\left(I, L_{2}(\partial \Omega)\right)$ and $\partial_{t} g \in L_{2}\left(I, L_{2}\left(\partial \Omega_{1}\right)\right)$. Then, the estimate $\left\|\nabla u_{i}\right\|_{0} \leq c$ holds for $i=1, \ldots, n$, for all $n$. 
Proof. We put $w=u_{i} \tau$ in (15) where we replace $f\left(\lambda, u_{i-1}, v_{i-1}\right)$ by $\delta v_{i}:=\frac{v_{i}-v_{i-1}}{\tau}$. Then we sum up for $i=1, \ldots, j$. We split $u_{i}-u_{i-1} \circ \varphi_{i}=u_{i}-u_{i-1}+u_{i-1}-u_{i-1} \circ \varphi_{i}$ and we use

$$
\frac{u_{i-1}-u_{i-1} \circ \varphi_{i}}{\tau}=\int_{0}^{1} \nabla \tilde{u}_{i-1}\left(x+s\left(\varphi_{i}(x)-x\right)\right) \mathrm{d} s . \omega_{h} * \frac{\bar{q}_{i}}{\theta_{i}},
$$

with $\left\|\omega_{h} * \frac{\bar{q}_{i}}{\theta_{i}}\right\|_{\infty} \leq c$. Due to the prolongation properties of $\tilde{u}_{i-1}$ and of Lemma 2.2 we have

$$
\left\|\frac{u_{i-1}-u_{i-1} \circ \varphi_{i}}{\tau}\right\|_{0} \leq c\left\|\nabla \tilde{u}_{i-1}\right\|_{L_{2}\left(\Omega^{*}\right)} \leq c\left\|\nabla u_{i-1}\right\|_{0}
$$

Then, we obtain

$$
\begin{gathered}
\frac{1}{2}\left(\left\|u_{j}\right\|_{0}^{2}-\left\|u_{0}\right\|_{0}^{2}\right)+\sum_{i=1}^{j}\left\|\nabla u_{i}\right\|_{0}^{2} \tau \leq c \sum_{i=1}^{j}\left\|\nabla u_{i}\right\|_{0}\left\|u_{i}\right\| \tau+\sum_{i=1}^{j}\left(\left\|h_{i}\right\|_{\partial \Omega_{1}}\right. \\
\left.+\left\|g_{i}\right\|_{\partial \Omega_{1}}\right)\left\|u_{i}\right\| \tau+\sum_{i=1}^{j} c\left\|u_{i}\right\|_{0} \tau+\sum_{i=1}^{j}\left\|G_{i}\right\|_{0}\left\|u_{i}\right\|_{0} \tau
\end{gathered}
$$

where (16), (18), the boundedness of $f$ and the continuous imbedding $V \hookrightarrow L_{2}(\partial \Omega)$ have been used. Young's inequality $\left(a b \leq \frac{\varepsilon^{2}}{2} a^{2}+\frac{1}{2 \varepsilon^{2}} b^{2}\right)$ and Gronwall's argument then lead to the a priori estimate

$$
\left\|u_{j}\right\|_{0}^{2}+\sum_{i=1}^{j}\left\|u_{i}\right\|^{2} \tau \leq c \quad \text { uniformly for } j, n .
$$

Next, we put $w=u_{i}-u_{i-1}$ in (14). In the surface integrals we apply Abel's summation and obtain the estimate

$$
\begin{aligned}
\theta_{0} \sum_{i=1}^{j}\left\|\delta u_{i}\right\|_{0}^{2} \tau+\frac{1}{2} c_{e}\left(\left\|\nabla u_{j}\right\|_{0}^{2}-\left\|\nabla u_{0}\right\|_{0}^{2}\right) \leq & c \sum_{i=1}^{j}\left\|\nabla u_{i}\right\|_{0}\left\|\delta u_{i}\right\|_{0} \tau+\left(h_{j}, u_{j}\right)_{\partial \Omega_{1}}-\left(g_{j}, u_{j}\right)_{\partial \Omega_{1}} \\
& +c \sum_{i=1}^{j}\left(\left\|\delta g_{i}\right\|_{\partial \Omega_{1}}+\left\|\delta h_{i}\right\|_{\partial \Omega_{1}}\right)\left\|u_{i}\right\| \tau \\
& +c \int_{\Lambda} \sum_{i=1}^{j}\left\|\delta v_{i}\right\|_{0}\left\|\delta u_{i}\right\|_{0} \tau \mathrm{d} \lambda+\sum_{i=1}^{j}\left\|G_{i}\right\|_{0}\left\|\delta u_{i}\right\|_{0} \tau,
\end{aligned}
$$

where we have again used the continuous imbedding $V \hookrightarrow L_{2}(\partial \Omega)$. Furthermore, we have

$$
\left|\left(h_{j}, u_{j}\right)_{\partial \Omega_{1}}\right| \leq\left\|h_{j}\right\|_{\partial \Omega}\left\|u_{j}\right\|_{\partial \Omega} \leq c_{1}\left\|h_{j}\right\|_{\partial \Omega_{1}}^{2}+\varepsilon\left\|\nabla u_{j}\right\|_{0}^{2}+c_{\varepsilon}\left\|u_{j}\right\|_{0}^{2}
$$

on account of the inequality -see [24],

$$
\|u\|_{\partial \Omega}^{2} \leq \varepsilon\|\nabla u\|_{0}^{2}+c_{\varepsilon}\|u\|_{0}^{2} \quad \forall u \in V, \varepsilon>0 \text { arbitrary small. }
$$

Similarly, we estimate $\left|\left(g_{j}, u_{j}\right)_{\partial \Omega_{1}}\right|$. Then, we apply (19) and Gronwall's argument and obtain

$$
\left\|\nabla u_{j}\right\|_{0}^{2} \leq c_{1}+c_{2} \sum_{i=1}^{j} \tau \int_{\Lambda}\left\|\delta v_{i}\right\|_{0}^{2} \mathrm{~d} \lambda \leq c,
$$


since $f$ is bounded and since $(10)_{2}$ implies that

$$
\left\|\int_{\Lambda} \delta v_{i} \mathrm{~d} \lambda\right\|_{0} \leq \int_{\Lambda}\left\|\delta v_{i}\right\|_{0} \mathrm{~d} \lambda \leq c .
$$

Thus, the proof is complete.

Remark 3.3. The estimate of $\left\|u_{i}\right\|_{C^{0, \alpha}},(i=1, \ldots, n)$, uniformly for $n$ is an open problem for $N>1$.

\section{Convergence of the Schemes I And II}

First, we derive some a priori estimates for $u_{i}, v_{i}$ and then we prove the convergence $\bar{u}^{n}(t) \rightarrow u(t), \bar{v}^{n}(t) \rightarrow$ $v(t)$ for $n \rightarrow \infty$ in corresponding functional spaces, where

and

$$
\bar{u}^{n}(t):=u_{i} \quad \text { for } \quad t \in\left(t_{i-1}, t_{i}\right), \quad i=1, \ldots, n, \quad \bar{u}^{n}(0)=0
$$

$$
u^{n}(t):=u_{i-1}+\left(t-t_{i-1}\right) \delta u_{i} \quad \text { for } t \in\left[t_{i-1}, t_{i}\right], i=1, \ldots, n
$$

and similarly we define $\bar{v}^{n}(t), v^{n}(t)$. We denote $\Phi_{n}(s):=\Psi_{n}(s) s-\int_{0}^{s} \Psi_{n}(z) \mathrm{d} z$ and similarly introduce $\Phi(s)$.

Lemma 4.1. Under the assumptions $\left.H_{1}\right)-H_{6}$ ) the following estimates hold:

$$
\begin{aligned}
& \max _{1 \leq i \leq n}\left\|u_{i}\right\|_{0}^{2} \leq c, \quad \max _{1 \leq i \leq n} \int_{\Omega} \Phi_{n}\left(u_{i}\right) \mathrm{d} x \leq c, \\
& \sum_{i=1}^{n}\left\|u_{i}\right\|^{2} \tau \leq c, \quad\left\|v_{i}\right\|_{0} \leq c, \quad \int_{\Lambda}\left\|\delta v_{i}\right\|_{0} \mathrm{~d} \lambda \leq c .
\end{aligned}
$$

Proof. We put $w=u_{i} \tau$ in (14) and sum up for $i=1, \ldots, j$. We denote the corresponding terms in the form $\sum_{l=1}^{6} J_{l}=J_{7}$. To estimate the term $J_{1}$ we use the splitting

$$
u_{i}-u_{i-1} \circ \varphi_{i}=u_{i}-u_{i-1}+u_{i-1}-u_{i-1} \circ \varphi_{i}
$$

and in $J_{2}$ we use

$$
\mu_{i}\left(u_{i}-u_{i-1}\right)=\Psi_{n}\left(u_{i}\right)-\Psi_{n}\left(u_{i-1}\right)+\tau \chi_{i}\left(u_{i}-u_{i-1}\right),
$$

with $\left\|\chi_{i}\right\|_{\infty} \leq c$ because of (11). Then using $H_{1}$ ) and the monotonicity of $\Psi_{n}$ and proceeding similarly as in Lemma 3.2 we get

$$
\begin{aligned}
J_{1}+J_{2} \geq & \frac{1}{2}\left(\left\|\theta_{j}^{1 / 2} u_{j}\right\|_{0}^{2}-\left\|\theta_{0}^{1 / 2} u_{0}\right\|_{0}^{2}\right)+\int_{\Omega} \Phi_{n}\left(u_{j}\right) \mathrm{d} x-\int_{\Omega} \Phi_{n}\left(u_{0}\right) \mathrm{d} x \\
& -c \sum_{i-1}^{j}\left\|u_{i}\right\|_{0}^{2} \tau-\sum_{i=1}^{j}\left(\theta_{i}\left(u_{i-1}-u_{i-1} \circ \varphi_{i}\right), u_{i}\right),
\end{aligned}
$$

since

$$
\sum_{i=1}^{j}\left(\theta_{i}\left(u_{i}-u_{i-1}\right), u_{i}\right) \geq \int_{\Omega} \frac{1}{2}\left(\theta_{i} u_{i}^{2}-\theta_{i-1} u_{i-1}^{2}\right) \mathrm{d} x+\frac{1}{2} \sum_{i-1}^{j} \int_{\Omega}\left(\theta_{i}-\theta_{i-1}\right) u_{i-1}^{2} \mathrm{~d} x
$$


and

$$
\begin{aligned}
\sum_{i-1}^{j}\left(\Psi_{n}\left(u_{i}\right)-\Psi_{n}\left(u_{i-1}\right), u_{i}\right) & \geq \int_{\Omega}\left(\Psi_{n}\left(u_{j}\right) u_{j}-\Psi_{n}\left(u_{0}\right) u_{0}\right) \mathrm{d} x-\sum_{i=1}^{j} \int_{\Omega}\left(Z_{n}\left(u_{i}\right)-Z_{n}\left(u_{i-1}\right)\right) \mathrm{d} x \\
& \geq \int_{\Omega} \Phi_{n}\left(u_{j}\right) \mathrm{d} x-\int_{\Omega} \Phi_{n}\left(u_{0}\right) \mathrm{d} x,
\end{aligned}
$$

where

$$
Z_{n}(s)=\int_{0}^{s} \Psi_{n}(r) \mathrm{d} r
$$

(see [17]). The last term in (22) is estimated by means of (16) and (18). We get

$$
\begin{gathered}
\left|\sum_{i=1}^{j}\left(\theta_{i}\left(u_{i}-u_{i-1} \circ \varphi_{i}\right), u_{i}\right)\right| \leq c_{\varepsilon} \sum_{i=1}^{j}\left\|u_{i}\right\|_{0}^{2} \tau \\
+\varepsilon \sum_{i=1}^{j}\left\|\nabla \tilde{u}_{i-1}\right\|_{L_{2}\left(\Omega^{*}\right)}^{2} \tau \leq \\
\varepsilon \sum_{i=1}^{j}\left\|\nabla u_{i}\right\|_{0}^{2} \tau+c_{\varepsilon} \sum_{i=1}^{j}\left\|u_{i}\right\|_{0}^{2} \tau .
\end{gathered}
$$

Using the boundedness of $f$ we find

$$
\left|J_{3}\right| \leq c_{1}+c_{2} \sum_{i=1}^{j}\left\|u_{i}\right\|_{0}^{2} \tau .
$$

Due to $\left.H_{1}\right)$ and (20) we have

$$
\begin{gathered}
\left|J_{4}\right| \leq c_{1} \sum_{i=1}^{j} \tau \int_{\partial \Omega} u_{i}^{2} \mathrm{~d} x+c_{2} \leq \varepsilon \sum_{i=1}^{j}\left\|\nabla u_{i}\right\|_{0}^{2} \\
+c_{\varepsilon} \sum_{i=1}^{j}\left\|u_{i}\right\|_{0}^{2} \tau+c .
\end{gathered}
$$

Similarly, we obtain

$$
\left|J_{5}\right| \leq \varepsilon \sum_{i=1}^{j}\left\|u_{i}\right\|_{0}^{2} \tau+c_{\varepsilon} \sum_{i=1}^{j}\left\|u_{i}\right\|_{0}^{2} \tau .
$$

Furthermore, $H_{2}$ ) leads to

$$
J_{6} \geq c_{\varepsilon} \sum_{i=1}^{j}\left\|u_{i}\right\|_{0}^{2} \tau .
$$

Finally, $H_{4}$ ) implies that

$$
\left|J_{7}\right| \leq c_{1}+c_{2} \sum_{i=1}^{j}\left\|u_{i}\right\|_{0}^{2} \tau .
$$


Combining (22-29) and again invoking Gronwall's argument we arrive at the desired a priori estimates.

Additional a priori estimates are found in the following lemma.

Lemma 4.2. The estimates

$$
\sum_{j-1}^{n-k}\left(b\left(t_{j+k}, u_{j+k}\right)-b\left(t_{j}, u_{j}\right), u_{j+k}-u_{j}\right) \tau \leq c k \tau, \quad 0 \leq k<n
$$

hold uniformly for $n$.

Proof. We rewrite (14) in the form

$$
\begin{gathered}
\left(b\left(t_{i}, u_{i}\right)-b\left(t_{i-1}, u_{i-1}\right), w\right)=-\left(D_{i} \nabla u_{i}, \nabla w\right) \tau \\
-\left(\theta_{i}\left(u_{i-1}-u_{i-1} \circ \varphi_{i}\right), w\right)+\left(u_{i-1}\left(\theta_{i}-\theta_{i-1}\right), w\right)+\left(h_{i} u_{i}, w\right)_{\partial \Omega_{1}} \tau \\
-\left(g_{i}, w\right)_{\partial \Omega_{1}} \tau+\left(G_{i}, w\right) \tau-\tau\left(\chi_{i}\left(u_{i}-u_{i-1}\right), w\right) \\
-\tau \int_{\Lambda}\left(f\left(\lambda, u_{i}, v_{i-1}\right), w\right) \mathrm{d} \lambda
\end{gathered}
$$

We sum up for $i=j+1, \ldots, j+k$. Then we put $w=\left(u_{j+k}-u_{j}\right) \tau$ and $\operatorname{sum}$ up for $j=1, \ldots, n-k$. In the second term on R.H.S. we use (16) and (18). Applying the a priori estimates of Lemma 4.1 we successively obtain the required estimate.

Corollary 4.3. As a special case of Lemma 4.2 we have

$$
\sum_{i=1}^{n}\left(u_{j+k}-u_{j}, u_{j+k}-u_{j}\right) \tau \leq c k \tau
$$

Proof. For the proof we take into account the monotonicity of $\Psi_{n}$. We get

$$
\left(\Psi_{n}\left(u_{j+k}\right)-\Psi_{n}\left(u_{j}\right), u_{j+k}-u_{j}\right) \geq 0 .
$$

Moreover, the rearrangement

$$
\theta_{j+k} u_{j+k}-\theta_{j} u_{j}=\theta_{j+k}\left(u_{j+k}-u_{j}\right)+\left(\theta_{j+k}-\theta_{j}\right) u_{j}
$$

implies that

$$
\sum_{j=i}^{n-k} \tau\left(\theta_{j+k} u_{j+k}-\theta_{j} u_{j}, u_{j+k}-u_{j}\right) \geq c \sum_{j=1}^{n-k}\left\|u_{j+k}-u_{j}\right\|_{0}^{2} \tau-\tau c \sum_{j=1}^{n-k}\left\|u_{j}\right\|_{0}^{2} \tau
$$

Then, from Lemmas 4.1 and 4.2 we obtain (31).

The a priori estimates in Lemma 4.1 and (31) can be rewritten in the form

$$
\begin{gathered}
\left\|\bar{u}^{n}(t)\right\|_{0} \leq c, \quad \int_{I}\left\|\bar{u}^{n}\right\|^{2} \mathrm{~d} t \leq c, \quad \max _{t} \int_{\Omega} \Phi_{n}\left(\bar{u}^{n}(t)\right) \mathrm{d} x \leq c, \\
\int_{I}\left\|\bar{u}^{n}(t+z)-\bar{u}^{n}(t)\right\|_{0}^{2} \mathrm{~d} t \leq c z \quad \forall 0<z \leq z_{0},
\end{gathered}
$$


where $k \tau \leq z \leq(k+1) \tau, \bar{u}^{n}(t+z)=0$ for $t+z>T$.

From $(32)_{2}$ we obtain (see [24])

$$
\int_{I} \int_{\Omega}\left(\bar{u}^{n}(t, x+y)-\bar{u}^{n}(t, x)\right)^{2} \mathrm{~d} x \mathrm{~d} t \leq c|y| \int_{I}\left\|\nabla \bar{u}^{n}\right\|_{0}^{2} \mathrm{~d} t \leq c|y| .
$$

Then, due to Kolmogoroff compactness argument (see [24]) we have

Lemma 4.4. There exists $u \in L_{2}(I, V)$ and a subsequence of $\left\{\bar{u}^{n}\right\}$ (we denote it again by $\left\{\bar{u}^{n}\right\}$ ) such that $\bar{u}^{n} \rightarrow$ $u$ in $L_{2}\left(I, L_{2}\right), \bar{u}^{n} \rightarrow u$ in $L_{2}(I, V)$. Moreover, there exists $v \in L_{2}\left(I, L_{2}(\Lambda \times \Omega)\right.$ such that $\partial_{t} v \in L_{2}\left(I, L_{2}(\Lambda \times \Omega)\right)$ and $\bar{v}^{n} \rightarrow v$ in $L_{2}\left(I, L_{2}(\Lambda \times \Omega)\right)$ for $n \rightarrow \infty$.

Proof. The convergence $\bar{u}^{n} \rightarrow u$ in $L_{2}\left(I, L_{2}(\Omega)\right)$ follows from Kolmogoroff's compactness argument (see [24]). From the a priori estimate $(32)_{2}$ we have that $\bar{u}^{n} \rightarrow w$ in $L_{2}(I, V)$ and also $\bar{u}^{n} \rightarrow w$ in $L_{2}\left(I, L_{2}\right)$. Consequently, $w \equiv u$. The boundedness of $f$ implies that

$$
\int_{\Lambda}\left\|\partial_{t} \bar{v}^{n}\right\|_{0} \mathrm{~d} \lambda \leq c \quad \text { where } \quad \partial_{t}^{\tau} \bar{v}^{n}:=\frac{v_{i}-v_{i-1}}{\tau} \quad \text { for } \quad t \in\left(t_{i-1}, t_{i}\right), i=1, \ldots, n .
$$

Then, $\partial_{t}{ }^{\tau} \bar{v}^{n}$ is bounded in $L_{2}\left(I, L_{2}(\Lambda \times \Omega)\right)$ and hence there exists $\chi \in L_{2}\left(I, L_{2}(\Lambda \times \Omega)\right)$ so that $\partial_{t}{ }^{\tau} \bar{v}^{n} \rightarrow \chi$ in $L_{2}\left(I, L_{2}(\Lambda \times \Omega)\right)$. We can write

$$
v^{n}(t)=v_{0}+\int_{0}^{t} f\left(\lambda, \bar{u}^{n}(s), \bar{v}_{\tau}^{n}(s)\right) \mathrm{d} s
$$

$\left(\bar{v}_{\tau}^{n}:=\bar{v}^{n}(t-\tau)\right.$ and $\bar{v}^{n}=v_{0}$ for $\left.s \in(-\tau, 0)\right)$. Using $\left.H_{4}\right)$ we get

$$
\begin{aligned}
\int_{\Lambda}\left\|v^{n}(t)-v^{m}(t)\right\|_{0}^{2} \mathrm{~d} \lambda & \leq \int_{\Lambda} \int_{0}^{t}\left\|f\left(\lambda, \bar{u}^{n}, \bar{v}_{\tau}^{n}\right)-f\left(\lambda, \bar{u}^{m}, \bar{v}_{\tau}^{m}\right)\right\|_{0}^{2} \mathrm{~d} s \mathrm{~d} \lambda \\
& \leq c \int_{\Lambda} \int_{0}^{t}\left(\left\|f\left(\lambda, \bar{u}^{n}, \bar{v}_{\tau}^{n}\right)-f\left(\lambda, \bar{u}^{m}, \bar{v}_{\tau}^{n}\right)\right\|_{0}^{2}+\left\|f\left(\lambda, \bar{u}^{m}, \bar{v}_{\tau}^{n}\right)-f\left(\lambda, \bar{u}^{m}, \bar{v}_{\tau}^{m}\right)\right\|_{0}^{2}\right) \mathrm{d} s \mathrm{~d} \lambda .
\end{aligned}
$$

Since $-K \leq f^{\prime}{ }_{v}() \leq$.0 we have

$$
\int_{\Lambda} \int_{0}^{t}\left\|f\left(\lambda, \bar{u}^{n}, \bar{v}_{\tau}^{n}\right)-f\left(\lambda, \bar{u}^{n}, \bar{v}_{\tau}^{m}\right)\right\|_{0}^{2} \mathrm{~d} s \mathrm{~d} \lambda \leq K \int_{\Lambda} \int_{0}^{t}\left\|\bar{v}^{n}-\bar{v}^{m}\right\|_{0}^{2} \mathrm{~d} s \mathrm{~d} \lambda .
$$

The boundedness of $\partial_{t} v^{n}$ in $L_{\infty}(I \times \Lambda \times \Omega)$ implies that $\left\|\bar{v}^{n}-\bar{v}^{n}\right\|_{0} \leq \frac{c}{n}$ (see (21)). Consequently, from Gronwall's argument we deduce that

$$
\int_{\Lambda}\left\|\bar{v}^{n}-\bar{v}^{m}\right\|_{0}^{2} \mathrm{~d} \lambda \leq c\left(\int_{0}^{t} \int_{\Lambda}\left\|f\left(\lambda, \bar{u}^{n}, \bar{v}_{\tau}^{n}\right)-f\left(\lambda, \bar{u}^{m}, \bar{v}_{\tau}^{n}\right)\right\|_{0}^{2} \mathrm{~d} s \mathrm{~d} \lambda+\frac{1}{n^{2}}+\frac{1}{m^{2}}\right) \mathrm{e}^{c K} .
$$

The R.H.S. converges to 0 for $n, m \rightarrow \infty$ since

$$
\left|f\left(\lambda, x, s_{n}, \xi\right)-f\left(\lambda, x, s_{m}, \xi\right)\right| \leq\left|f\left(\lambda, x, s_{n}, \xi\right)-f(\lambda, x, s, \xi)\right|+\left|f\left(\lambda, x, s_{m}, \xi\right)-f(\lambda, x, s, \xi)\right|
$$

and since $f\left(\lambda, x, s_{n}, \xi\right) \rightarrow f(\lambda, s, \xi)$ uniformly with respect to $\xi$ in bounded sets.

As $\bar{v}_{\tau}^{n}$ is bounded and $f$ is bounded, we obtain that $\bar{v}^{n} \rightarrow v$ in $L_{2}\left(I, L_{2}(\Lambda \times \Omega)\right.$ ) (moreover in $L_{\infty}\left(I, L_{2}(\Lambda \times \Omega)\right)$ ) because of Lebesgue dominated convergence theorem. From this fact and from $\partial_{t}{ }^{\tau} \bar{v}_{n} \rightarrow \chi$ it follows that $\partial_{t} v=\chi$ and Lemma 4.4 is proved.

Now we can prove our main result. Let $\{\bar{n}\}$ denote a suitable subsequence of $\{n\}$. 
Theorem 4.5. Let the assumptions $\left.\left.H_{1}\right)-H_{6}\right)$ be satisfied. Then $\{u, v\}$ from Lemma 4.4 is a variational solution of (1)-(4) in the sense of Definition 1. The approximate solution $\left\{\bar{u}^{\bar{n}}, \bar{v}^{\bar{n}}\right\}$ from (7-9), (resp. (10-9)), converges to $\{u, v\}: \quad \bar{u}^{\bar{n}} \rightarrow u$ in $L_{2}(I, V)$ and $\bar{v}^{\bar{n}} \rightarrow v$ in $L_{\infty}\left(I, L_{2}(\Lambda \times \Omega)\right)$. If, moreover, $f$ is nondecreasing in $u$ and (5) is satisfied with div $\bar{q} \in L_{\infty}\left(Q_{T}\right)$, then this solution is unique and the original sequence $\left\{\bar{u}^{n}, \bar{v}^{n}\right\}$ is convergent.

Proof. First we prove that $u$ from Lemma 4.4 is a variational solution of (1-4). By the duality argument in (30) and due to the a priori estimates (32) we obtain

$$
\left\|\delta b\left(t_{i}, u_{i}\right)\right\|_{*}=\sup _{\|w\| \leq 1}\left(\delta b\left(t_{i}, u_{i}\right), w\right) \leq c_{1}+c_{2}\left\|u_{i}\right\|
$$

Consequently, $\partial_{t}{ }^{\tau} b_{n}\left(t, \bar{u}^{n}\right)$ is bounded in $L_{2}\left(I, V^{*}\right)$, where $b_{n}\left(t, \bar{u}^{n}\right):=b\left(t_{i}, u_{i}\right)$ for $t \in\left(t_{i-1}, t_{i}\right), i=1, \ldots, n$. Thus $\partial_{t}^{\tau} b_{n}\left(t, \bar{u}^{n}\right) \rightarrow \chi$ in $L_{2}\left(I, V^{*}\right)$. On the other hand $\bar{u}^{n}(t, x) \rightarrow u(t, x)$ a.e. $(t, x) \in Q_{T}$. Due to the properties of $\theta$ and $\Psi_{n}$ (see $\left.H_{1}\right)$ and $\left.H_{6}\right)$ ) we obtain that $\Psi_{n}\left(\bar{u}^{n}\right) \rightarrow \Psi(u)$ and consequently $b_{n}\left(t, \bar{u}^{n}\right) \rightarrow b(t, u)$ for a.e. $(t, x) \in Q_{T}$. Moreover, the following estimate holds:

$$
\left|\Psi_{n}(s)\right| \leq \delta \Phi_{n}(s)+c_{\delta} \quad \text { for any } \quad \delta>0, \text { uniformly for } \quad s \in R
$$

which can be obtained in the same way as (4.7) in [17]. From this estimate and from $\int_{\Omega} \Phi_{n}\left(\bar{u}^{n}\right) \mathrm{d} x \leq c$ it follows that $\Psi_{n}\left(\bar{u}^{n}\right) \rightarrow \Psi(u)$ in $L_{1}\left(Q_{T}\right)$ and consequently that $b_{n}\left(t, \bar{u}^{n}\right) \rightarrow b(t, u)$ in $L_{1}\left(Q_{T}\right)$. From this fact we obtain $\chi \equiv \partial_{t} b(t, u)$. We rewrite $(30)$ in the form

$$
\begin{aligned}
\int_{I}\left(\partial_{t}{ }^{\tau} b_{n}\left(t, \bar{u}^{n}\right), w\right) \mathrm{d} t & -\int_{I}\left(\partial_{t}^{\tau} \theta \bar{u}_{\tau}^{n}, w\right) \mathrm{d} t+\int_{I}\left(\bar{D}^{n} \nabla \bar{u}^{n}, \nabla w\right) \mathrm{d} t \\
+A_{n}-\int_{I}\left(\bar{h}^{n} \bar{u}^{n}, w\right)_{\partial \Omega_{1}} \mathrm{~d} t & +\int_{I}\left(\bar{g}^{n}, w\right)_{\partial \Omega_{1}} \mathrm{~d} t+\int_{I} \int_{\Lambda}\left(f\left(\lambda, \bar{u}^{n}, \bar{v}_{\tau}^{n}\right), w\right) \mathrm{d} t \\
& =\int_{I}\left(\bar{G}^{n}, w\right) \mathrm{d} t-\int_{I}\left(\bar{\chi}^{n}\left(\bar{u}^{n}-\bar{u}_{\tau}^{n}\right), w\right) \mathrm{d} t, \quad \forall w \in L_{2}(I, V),
\end{aligned}
$$

where

$$
A_{n}=\int_{I}\left(\bar{\theta}^{n} \frac{1}{\tau}\left(\bar{u}_{\tau}^{n}-\bar{u}_{\tau}^{n} \circ \varphi^{n}\right), w\right) \mathrm{d} t
$$

$\bar{D}^{n}=D\left(t_{i}, x\right)$ for $t \in\left(t_{i-1}, t_{i}\right), i=1, \ldots, n$, and similarly for $\bar{\theta}^{n}, \bar{\chi}^{n}, \bar{G}^{n}, \bar{g}^{n}, \bar{h}^{n}, \bar{\varphi}^{n}$.

Next we prove that $A_{n} \rightarrow \int_{I}(\bar{q} . \nabla u, w) \mathrm{d} t$. For this purpose we use (16) and the convergence property $\omega_{h} * \frac{\bar{q}^{n}}{\theta^{n}} \rightarrow \frac{\bar{q}}{\theta}$ for $n \rightarrow \infty$ in $L_{2}\left(Q_{T}\right)$ and also pointwise a.e. in $(t, x) \in Q_{T}$. Moreover, we show that

$$
\int_{0}^{1} \nabla \overline{\tilde{u}}_{\tau}^{n}\left(t, x+s\left(\bar{\varphi}^{n}(x)-x\right)\right) \mathrm{d} s \rightarrow \nabla u \quad \text { in } \quad L_{2}\left(I, L_{2}\right)
$$

as a consequence of (see (18))

$$
\int_{I}\left\|\nabla \int_{0}^{1} \overline{\tilde{u}}^{n}(.) \mathrm{d} s\right\|_{0}^{2} \mathrm{~d} t \leq c \int_{I}\left\|\nabla \bar{u}^{n}\right\|_{0}^{2} \mathrm{~d} t \leq c
$$

and

$$
\int_{0}^{1} \overline{\tilde{u}}_{\tau}^{n}(.) \mathrm{d} s-\bar{u}_{\tau}^{n}=\tau \omega_{h} * \frac{\bar{q}^{n}}{\theta^{n}} \cdot \int_{0}^{1} \int_{0}^{1} \nabla \tilde{\bar{u}}_{\tau}^{n}\left(t, x+s r\left(\bar{\varphi}^{n}(x)-x\right)\right) \mathrm{d} r \mathrm{~d} s .
$$


Notice that (36) implies that $\nabla \int_{0}^{1} \overline{\tilde{u}}^{n} \rightarrow \chi \equiv \nabla u$ in $L_{2}\left(I, L_{2}\right)$ since $\bar{u}_{\tau}^{n} \rightarrow u$ and consequently $\int_{0}^{1} \overline{\tilde{u}}_{\tau}^{n}(.) \mathrm{d} s \rightarrow u$ in $L_{2}\left(I, L_{2}\right)$. The boundedness and pointwise convergence of $\omega_{h} * \frac{\bar{q}^{n}}{\theta^{n}}$ then guarantee that $A_{n} \rightarrow \int_{I}(\bar{q} . \nabla u, w) \mathrm{d} t$ for $n \rightarrow \infty$. From $\bar{u}^{n} \rightarrow u$ in $L_{2}\left(I, L_{2}\right)$ and $\bar{u}^{n} \rightarrow u$ in $L_{2}(I, V)$, it follows that $\bar{u}^{n} \rightarrow u$ in $L_{2}\left(I, L_{2}(\partial \Omega)\right)-$ see $[18]$.

Now, we can take the limit $n \rightarrow \infty$ in (35) and obtain the identity ii) in Definition 2.1. To prove identity iii) we consider

$$
\begin{aligned}
\int_{I}\left\langle\frac{b(t, u(t))-b(t-\tau, u(t-\tau))}{\tau}, \zeta\right\rangle \mathrm{d} t= & \frac{1}{\tau} \int_{I}(b(t, u(t))-b(t-\tau, u(t-\tau)), \zeta) \mathrm{d} t \\
= & -\int_{0}^{T-\tau}\left(b(t, u(t)), \frac{\zeta(t-\tau)-\zeta(t)}{\tau}\right) \mathrm{d} t \\
& -\frac{1}{\tau} \int_{T-\tau}^{T}(b(t, u(t)), \zeta(t)) \mathrm{d} t-\frac{1}{\tau} \int_{0}^{\tau}\left(b\left(0, u_{0}\right), \zeta(s)\right) \mathrm{d} s
\end{aligned}
$$

where $b(t, u(t)) \equiv b\left(0, u_{0}\right)$ for $t \in(-\tau, 0)$. Next, we take the limit $\tau \rightarrow 0$ and use $\frac{1}{\tau}\{b(t, u(t)-b(t-\tau, u(t-$ $\tau))\} \rightarrow \partial_{t} b(t, u)$ in $L_{2}\left(I, V^{*}\right)$. We conclude that $\{u, v\}$ from Lemma 4.4 is a variational solution of (1-4). The convergence of the original sequences $\left\{\bar{u}^{n}, \bar{v}^{n}\right\}$ is a consequence of the uniqueness result in [21].

In fact, we can prove a stronger convergence $\bar{u}^{n} \rightarrow u$ than in Theorem 4.5.

Theorem 4.6. Under the assumptions of Theorem 4.5 it holds that $\bar{u}^{n} \rightarrow u$ in $L_{2}(I, V)$.

Proof. In the proof we essentially make use of the integration by parts formula - see $[1,17]$

$$
\begin{aligned}
\int_{0}^{t}\left\langle\partial_{t} b(t, u), u\right\rangle \mathrm{d} t= & \int_{\Omega} B(t, u(t)) \mathrm{d} x-\int_{\Omega} B\left(0, u_{0}\right) \mathrm{d} x \\
& +\frac{1}{2} \int_{0}^{t} \int_{\Omega} \partial_{t} \theta u^{2} \mathrm{~d} x \mathrm{~d} t
\end{aligned}
$$

where $B(t, u)=\frac{1}{2} \theta u^{2}+\Phi(u)$ and $\nabla \Phi=\Psi$ is from Section 3 .

We note that $\int_{\Omega} \Phi(u(t)) \mathrm{d} z \leq c<\infty$ follows from a priori estimates in Lemma 4.1 and from Fatou's lemma $(\Phi(s) \geq 0$ is convex). Moreover, we shall use

$$
\liminf \int_{0}^{t}\left(\partial_{t}{ }^{\tau} b_{n}\left(t, \bar{u}^{n}\right), \bar{u}^{n}\right) \mathrm{d} t \geq \int_{\Omega} B(t, u(t)) \mathrm{d} x-\int_{\Omega} B\left(0, u_{0}\right) \mathrm{d} x+\frac{1}{2} \int_{0}^{t} \int_{\Omega} \partial_{t} \theta u^{2} \mathrm{~d} x \mathrm{~d} t,
$$

which we obtain from the following inequality (see $(22,23))$

$$
\begin{gathered}
\sum_{i=1}^{j}\left\{\left(\theta_{i} u_{i}-\theta_{i-1} u_{i-1}, u_{i}\right)+\left(\Psi_{n}\left(u_{i}\right)-\Psi\left(u_{i-1}\right), u_{i}\right)\right\} \geq \\
\int_{\Omega}\left\{\frac{1}{2} \theta_{j} u_{j}^{2}+\Phi_{n}\left(u_{j}\right)\right\} \mathrm{d} x-\int_{\Omega}\left\{\frac{1}{2} \theta_{0} u_{0}^{2}+\Phi_{n}\left(u_{0}\right)\right\} \mathrm{d} x+\frac{1}{2} \sum_{i=1}^{j} \tau \int_{\Omega} \frac{\theta_{i}-\theta_{i-1}}{\tau} u_{i-1}^{2} \mathrm{~d} x \\
+\sum_{i=1}^{j} \tau \int_{\Omega} \frac{\theta_{i}-\theta_{i-1}}{\tau}\left(u_{i-1} u_{i}-u_{i-1}^{2}\right) \mathrm{d} x .
\end{gathered}
$$

The last term converges to 0 for $n \rightarrow \infty$. This is seen as follows. Firstly, $\frac{\theta_{i}-\theta_{i-1}}{\tau} \equiv \partial_{t} \theta^{n}(t, x)$ for $t \in\left(t_{i-1}, t_{i}\right)$ is bounded and converges to $\partial_{t} \theta(t, x)$ for $n \rightarrow \infty$ for a.e. $(t, x) \in Q_{T}$. Secondly, $\bar{u}_{\tau}^{n} \bar{u}^{n}-\left(\bar{u}_{\tau}^{n}\right)^{2} \rightarrow u^{2}-u^{2}=0$ 
for a.e. $(t, x) \in Q_{T}$. In an analogous way we obtain

$$
\frac{1}{2} \sum_{i=1}^{j} \tau \int_{\Omega} \frac{\theta_{i}-\theta_{i-1}}{\tau} u_{i-1}^{2} \mathrm{~d} x \rightarrow \frac{1}{2} \int_{0}^{t} \int_{\Omega} \partial_{t} \theta u^{2} \mathrm{~d} x \mathrm{~d} t \quad \text { for } \quad n \rightarrow \infty
$$

provided that $t_{j} \equiv t_{j}^{n} \rightarrow t$.

From (38) and (39) we deduce that

$$
\liminf \int_{0}^{t}\left\langle\delta_{t}^{\tau} b_{n}\left(t, \bar{u}^{n}\right), \bar{u}^{n}-u\right\rangle \mathrm{d} t \geq 0
$$

since $\partial_{t}{ }^{\tau} b_{n}\left(t, \bar{u}^{n}\right) \rightarrow \partial_{t} b(t, u)$ in $L_{2}\left(I, V^{*}\right)$ - see the proof of Theorem 4.5.

Next, we put $w=\bar{u}^{n}-u$ in (35) and we use (40) and the estimate

$$
\begin{aligned}
\int_{0}^{t}\left(\bar{D}^{n} \nabla \bar{u}^{n}, \nabla\left(\bar{u}^{n}-u\right)\right) \mathrm{d} t & =\int_{0}^{t}\left(\bar{D}^{n} \nabla\left(\bar{u}^{n}-u\right), \nabla\left(\bar{u}^{n}-u\right)\right) \mathrm{d} t+\int_{0}^{t}\left(\bar{D}^{n} \nabla u, \nabla\left(\bar{u}^{n}-u\right)\right) \mathrm{d} t \\
& \geq c \int_{0}^{t}\left\|\nabla\left(\bar{u}^{n}-u\right)\right\|_{0}^{2} \mathrm{~d} t+\varepsilon_{n},
\end{aligned}
$$

where

since $\bar{u}^{n} \rightarrow u$ in $L_{2}(I, V)$.

In the remaining terms of (35) we use the fact that $\bar{u}^{n} \rightarrow u$ in $L_{2}\left(I, L_{2}(\Omega)\right)$ and $\bar{u}^{n} \rightarrow u$ in $L_{2}\left(I, L_{2}(\partial \Omega)\right)$. Then, the required convergence result follows.

\section{Some Generalizations}

In this section we consider a more general case of reaction-adsorption term $f$. Instead of $\left.H_{4}\right)$ we assume $\left.H_{4}\right)^{\prime}: f(\lambda, x, z, w)$ is continuous on $\Lambda \times R \times R$ and satisfies:

1) $|f(\lambda, x, z, w)| \leq c(1+|z|+|w|)$,

2) $-K \leq{f^{\prime}}_{w}(\lambda, x, z, w) \leq 0$ for $(\lambda, x, z, w) \in \Lambda \times \Omega \times \Omega \times R$ and

$3)$ if $z_{n} \rightarrow z$ then $f\left(\lambda, x, z_{n}, \xi\right) \rightarrow f(\lambda, x, z, \xi)$ uniformly in $\lambda \in \Lambda, \xi \in R$ Assumption 3$)$ is evidently satisfied for

$$
f(\lambda, x, u, v)=k(\lambda, x)(\varphi(\lambda, u)-v)
$$

with $k(\lambda, x) \leq M$. The uniform boundedness of $k(\lambda, x)$ is implicitly included in 1$)$.

Theorem 5.1. If we replace $\left.\mathrm{H}_{4}\right)$ by $\left.\mathrm{H}_{4}\right)^{\prime}$ then the results of Theorems 4.5 and 4.6 remain valid.

Proof. For the proof we must only include the analysis of the reactive term concerning $f$. In the proof of Lemma 4.1 we estimate (we omit $x$ in $f$ )

$$
\left|\sum_{i=1}^{j}\left(\int_{\Lambda} f\left(\lambda, u_{i}, v_{i-1}\right) \mathrm{d} \lambda, u_{i}\right) \tau\right| \leq c_{1}+c_{2} \sum_{i=1}^{j}\left\|u_{i}\right\|_{0}^{2} \tau+c_{3} \sum_{i=1}^{j} \tau \int_{\Lambda}\left\|v_{i}\right\|_{0}^{2} \mathrm{~d} \lambda .
$$

$\left.\left(\text { see } H_{4}\right)^{\prime}\right)$. For the last term we notice that

$$
v_{i}=v_{i-1}+\tau\left\{f\left(\lambda, x, u_{i-1}, 0\right)+{f^{\prime}}_{v}\left(\lambda, x, u_{i}, \xi_{i}\right) v_{i-1}\right\} \quad \text { for a.e. } \lambda \in \Lambda, x \in \Omega
$$


where $\xi_{i}=\xi_{i}(x, \lambda) \in\left(0, v_{i-1}\right)$. Consequently,

$$
\left|v_{i}\right| \leq\left\{1+\tau f^{\prime}{ }_{v}(.)\right\} v_{i-1}+\left|F\left(\lambda, u_{i}, 0\right)\right| \tau,
$$

where $0<q_{i}:=1+\tau f^{\prime}{ }_{v}()<$.1 for $\tau \leq \tau_{0}$.

From this recurrent inequality we obtain

$$
\left|v_{i}\right| \leq Q_{1} v_{0}+\sum_{l=1}^{i} Q_{i-l}\left|f\left(\lambda, x, u_{l}, 0\right)\right| \tau
$$

with $Q_{i-l}=\prod_{j=l}^{i} q_{j}$. This leads to

$$
\int_{\Lambda}\left\|v_{i}\right\|_{0}^{2} \mathrm{~d} \lambda \leq c_{1}+c_{2} \sum_{l=1}^{i}\left\|u_{l}\right\|_{0}^{2} \tau
$$

We insert this estimate in (41) and obtain

$$
\left|\sum_{i=1}^{j}\left(\int_{\Lambda} f\left(\lambda, u_{i}, v_{i-1}\right) \mathrm{d} \lambda, u_{i}\right) \tau\right| \leq c_{1}+c_{2} \sum_{i=1}^{j}\left\|u_{i}\right\|_{0}^{2} \tau,
$$

which will guarantee the a priori estimates of Lemma 4.1.

Consequently, from (42) we get

$$
\left\|v_{i}\right\|_{L_{2}(\Lambda \times \Omega)} \leq c \quad \text { and }\left\|\delta v_{i}\right\|_{L_{2}(\Lambda \times \Omega)} \leq c, \quad i=1, \ldots, n,
$$

uniformly for $n$. The assertion of Lemma 4.2 remains unchanged. In the proof of Lemma 4.4 we can use the same arguments because of the stronger assumption iii) in $H_{4}$ ), since we cannot guarantee the $L_{\infty}$ boundedness of $v_{i}$. Then, along the same lines as in Section 4 we obtain that the Theorems 4.5 and 4.6 remain valid.

The same results are obtained using the implicit approximation scheme (14), where we replace $f\left(\lambda, u_{i}, v_{i}\right)$ by $f\left(\lambda, u_{i-1}, v_{i}\right)$ or by $f\left(\lambda, u_{i-1}, v_{i-1}\right)$. In the first case we shall have

$$
\left(1+\tau f^{\prime}{ }_{v}(.)\right) v_{i}=v_{i-1}+\tau f\left(\lambda, u_{i}, 0\right),
$$

with $\left(1+\tau f^{\prime}{ }_{v}().\right) \geq 1$ (without the restriction $\tau \leq \tau_{0}$ ). Also in this case we obtain the estimate (42).

Remark 5.2. The convergence results can also be extended to the more general situation where 1) in $\left.H_{4}\right)^{\prime}$ is replaced by

$$
1)^{\prime} \quad|f(\lambda, x, z, w)| \leq c(1+F(z)+P(w))
$$

where $F$ and $P$ are nonnegative continuous functions. Indeed, in the case of a unique variational solution (see [21]) we can use the truncation method, since the concentrations $u$ and $v$ satisfy $0 \leq u, v \leq 1$. In that case we use $\bar{f}$ instead of $f$, where

$$
\bar{f}(\lambda, x, z, w)=f(\lambda, x, p(z), p(w)),
$$

with

$$
p(s)= \begin{cases}s & s \in[0,1] \\ 1 & s \geq 1 \\ 0 & s \leq 0 .\end{cases}
$$

Then, $\bar{f}$ is bounded and the arguments of Section 4 can be used. 


\section{Full Discretization scheme}

The convergence results obtained in Sections 4 and 5 remain valid for the full discretization scheme where (7), respectively (10), is solved in a finite dimensional space, e.g. by a FEM. Then, we shall look for $u_{i}^{\gamma} \in V_{\gamma} \subset V \equiv$ $W_{2}^{1}(\Omega)$, where $\operatorname{dim} V_{\gamma}<\infty$ and where $V_{\gamma} \rightarrow V$ for $\gamma \rightarrow 0$ in canonical sense. We determine $u_{i}^{\gamma} \equiv u_{i}^{\gamma, \delta}$ from $($ see $(14))$

$$
\begin{gathered}
\left(\theta_{i} \frac{u_{i}^{\gamma}-u_{i-1}^{\gamma} \circ \varphi_{i}}{\tau}, w\right)+\left(\varrho_{1} \mu_{i} \frac{u_{i}^{\gamma}-u_{i-1}^{\gamma}}{\tau}, w\right)+\sum_{k=1}^{M_{\delta}} \mu\left(E_{k}\right)\left(\varrho_{2} f\left(\lambda_{k}, u_{i}^{\gamma}, v_{i-1}^{\gamma, k}\right), w\right) \\
+\left(g_{i}, w\right)_{\partial \Omega_{1}}+\left(D_{i} \nabla u_{i}^{\gamma}, \nabla w\right)=\left(G_{i}, w\right), \quad \forall w \in V_{\gamma},
\end{gathered}
$$

for $i=1, \ldots, n$, where

$$
\frac{v_{i}^{\gamma, k}-v_{i-1}^{\gamma, k}}{\tau}=f\left(\lambda_{k}, u_{i}^{\gamma}, v_{i-1}^{\gamma, k}\right) \quad \text { for } \quad \lambda_{k} \in E_{k} \subset \Lambda, k=1, \ldots, M_{\delta} .
$$

We assume that $\left\{E_{k}\right\}_{1}^{M_{\delta}}$ are $\mu$-measurable $\left(E_{k} \equiv E_{k}^{\delta}\right)$ and that $\cup_{k=1}^{M_{\delta}} E_{k} \cup \Lambda_{0}=\Lambda$, with $\mu\left(\Lambda_{0}\right)=0$ and $E_{k} \bigcap E_{l}=\emptyset$ for $k \neq l$.

We define

$$
\bar{f}^{\delta}(\lambda, \xi, \eta):=f\left(\lambda_{k}, \xi, \eta\right) \text { for } \lambda \in E_{k}, k=1, \ldots, M_{\delta}
$$

Moreover, we assume that

$$
\bar{f}^{\delta}(\lambda, \xi, \eta) \rightarrow f(\lambda, \xi, \eta) \quad \text { for } \quad \delta \rightarrow 0, \mu \text { - a.e. in } \Lambda \text { uniformly for }(\xi, \eta) \text { in bounded sets. }
$$

Let $\alpha, \alpha \equiv(\tau, \gamma, \delta)$, represents the discretization parameter corresponding to the discretization of I, $\Omega$ and $\Lambda$, respectively.

By means of $u_{i}^{\gamma, \delta}$ and $v_{i}^{\gamma, \delta}$ we define Rothe's functions $\bar{u}^{\alpha}(t)$ and $\bar{v}^{\alpha}$ for $\alpha=(\tau, \gamma, \delta)$

$$
\bar{u}^{\alpha}(t):=u_{i}^{\gamma, \delta}, \quad \bar{v}^{\alpha}:=v_{i}^{k} \quad \text { for } t \in\left(t_{i-1}, t_{i}\right), i=1, \ldots, n, \lambda \in E_{k},
$$

where $\bar{u}^{\alpha}$ is independent on $\lambda$. Generally, $\bar{v}^{\alpha}$ doesn't belong to $V_{\gamma}$. Using the arguments from Section 4 we can prove the convergences $\bar{u}^{\alpha} \rightarrow u$ and $\bar{v}^{\alpha} \rightarrow v$ in corresponding functional spaces, where $\{u, v\}$ is a variational solution of (1-4).

Theorem 6.1. Under the assumptions of Theorem 4.5 the assumption (45) and the convergence $V_{\gamma} \rightarrow V$ for $\gamma \rightarrow 0$ (in the canonical sense) it holds that $\bar{u}^{\bar{\alpha}} \rightarrow u$ in $L_{2}(I, V), \bar{v}^{\bar{\alpha}} \rightarrow v$ in $L_{2}\left(I, L_{2}(\Lambda \times \Omega)\right)$ for $\bar{\alpha} \rightarrow 0$, where $\{u, v\}$ is a variational solution of (1-4) ( $\bar{\alpha}$ is a suitable subsequence of $\alpha$ ). If $f$ is nondecreasing in $u$, then the original sequences $\left\{\bar{u}^{\alpha}\right\}$ and $\left\{\bar{v}^{\alpha}\right\}$ are convergent.

Proof. We follow the arguments of Section 4 -see the proof of Theorem 4.5. The term

$$
\sum_{k=1}^{M_{\delta}} \mu\left(E_{k}\right)\left(\varrho_{2} f\left(\lambda_{k}, u_{i}^{\gamma}, v_{i-1}^{\gamma, k}\right), w\right)
$$

can be rewritten as

$$
\int_{\Lambda}\left(\varrho_{2} \bar{f}^{\delta}\left(\lambda, u_{i}^{\gamma}, \bar{v}_{i-1}^{\gamma, \delta}\right), w\right) \mathrm{d} \lambda
$$

where $v_{i-1}^{\gamma, \delta}(\lambda):=v_{i-1}^{\gamma, k}$ for $\lambda \in E_{k}, k=1, \ldots, M_{\delta}$. Without substantial changes we obtain similar a priori estimates as in Lemma 4.1, since we have formally the same mathematical model (just projected on $V_{\gamma}$ ). Also 
Lemmas 4.2 and 4.4 are obtained along the same lines. Unlike in Section 4 we do not have the boundedness of $\partial_{t}^{\tau} b_{n}\left(t, \bar{u}^{\alpha}\right)$ in $L_{2}\left(I, V^{*}\right)$, but only

$$
\left\|\partial_{t}^{\tau} b_{n}\left(t, \bar{u}^{\alpha}\right)\right\|_{L_{2}\left(I, V_{\gamma}^{*}\right)} \leq c
$$

In this case we can extend the functional $\partial_{t}^{\tau} b_{n}\left(t, \bar{u}^{\alpha}\right) \in L_{2}\left(I, V_{\gamma}^{*}\right)$ to $F_{n} \in L_{2}\left(I, V^{*}\right)$ by the following definition, (see [16])

$$
\begin{aligned}
\int_{I}\left\langle F_{n}, w\right\rangle \mathrm{d} t & :=\int_{I}\left\langle\partial_{t}^{\tau} b_{n}\left(t, \bar{u}^{\alpha}\right), P_{\gamma} \bar{w}^{\alpha}\right\rangle \mathrm{d} t \\
& =\int_{I} \int_{\Omega} \partial_{t}^{\tau} b_{n}\left(t, \bar{u}^{\alpha}\right) P_{\gamma} w \mathrm{~d} x \mathrm{~d} t
\end{aligned}
$$

where $P_{\gamma}: V \rightarrow V_{\gamma}$ is a projector. Then, $F_{n} \rightarrow F$ in $L_{2}\left(I, V^{*}\right)$, since $\left\|F_{n}\right\|_{L_{2}\left(I, V^{*}\right)} \leq c$. Next, $b_{n}\left(t, \bar{u}^{\alpha}\right) \rightarrow b(t, u)$ in $L_{1}\left(Q_{T}\right)$ implies that $F \equiv \partial_{t} b(t, u)$.

To prove that $\{u, v\}$ (from Th. 4.5) is a variational solution we use a test function $\bar{w}^{\alpha} \in L_{2}\left(I, V_{\gamma}\right)$ in the approximate equation (see (35)), where $\bar{w}^{\alpha} \rightarrow w$ in $L_{2}(I, V)$ for $\alpha \rightarrow 0$. Then, taking the limit $\alpha \rightarrow 0$ we get

$$
\int_{0}^{t}\left\langle\partial_{t}^{\tau} b_{n}\left(t, \bar{u}^{\alpha}\right), \bar{w}^{\alpha}\right\rangle \mathrm{d} t=\int_{0}^{t}\left\langle F_{n}, P_{\gamma} \bar{w}^{\alpha}\right\rangle \mathrm{d} t \rightarrow \int_{0}^{t}\langle F, w\rangle \mathrm{d} t=\int_{0}^{t}\left\langle\partial_{t} b(t, u), w\right\rangle \mathrm{d} t .
$$

In the reaction term we use the continuity of $f$ in its variables and the convergence $\bar{f}^{\delta}\left(\lambda, \bar{u}^{\alpha}, \bar{v}_{\tau}^{\alpha}\right) \rightarrow f(\lambda, u, v)$ for a.e. $\{\lambda,(t, x)\}$ in $\Lambda \times Q_{T}$ (with respect to the product measure $\mu \times \mathrm{d} x \mathrm{~d} t-$ see [13]).

To prove that $\bar{u}^{\alpha} \rightarrow u$ in $L_{2}(I, V)$ we use (38) and (39) (where we replace $\bar{u}^{n}$ by $\bar{u}^{\alpha}$ ) and (46), such that

$$
\liminf \int_{0}^{t} \int_{\Omega} \partial_{t}^{\tau} b_{n}\left(t, \bar{u}^{\alpha}\right)\left(\bar{u}^{\alpha}-\bar{w}^{\alpha}\right) \mathrm{d} x \mathrm{~d} t=\liminf \int_{0}^{t}\left\langle\partial_{t}^{\tau} b_{n}\left(t, \bar{u}^{\alpha}\right), \bar{u}^{\alpha}-\bar{w}^{\alpha}\right\rangle \mathrm{d} t \geq 0
$$

provided that $\bar{w}^{\alpha} \in L_{2}\left(I, V_{\gamma}\right)$, with $\bar{w}^{\alpha} \rightarrow u$ in $L_{2}(I, V)$. We can put $w=\bar{u}^{\alpha}-\bar{w}^{\alpha}$ in (35) where we replace $\bar{u}^{n}$ by $\bar{u}^{\alpha}$ and take liminf for $\alpha \rightarrow 0$. We proceed as in the proof of Theorem 4.5. Due to (47) and the convergences $\bar{u}^{\alpha} \rightarrow u$ in $L_{2}\left(I, L_{2}\right), \bar{u}^{\alpha} \rightarrow u$ in $L_{2}\left(I, L_{2}(\partial \Omega)\right.$ ), (see [18]), we deduce that $\bar{u}^{\alpha} \rightarrow u$ in $L_{2}(I, V)$, which implies the required result.

\section{Numerical implementation}

Our concept of numerical approximation of (1-4) takes into account the following facts: the degeneracy of the parabolic term $\partial_{t} \Psi(u)$; non-equilibrium adsorption in (2), with eventually non-Lipschitz continuity of $f$ with respect to $u$; dominance of the convective term represented by $\bar{q}$; and non-smoothness (in the space variables) of $\theta$ and $\bar{q}$.

The crucial problem represents the dominant convective term. We treat it by the method of characteristics, which suffers with the preservation of mass balance as mentioned in the introduction. Moreover, when applying a FEM, we have to evaluate the integrals

$$
\left(u_{i-1} \circ \varphi_{i}, \Phi_{j}\right)=\int_{\Omega} u_{i-1} \circ \varphi_{i} \Phi_{j} \mathrm{~d} x
$$

where $\Phi_{j}$ are the basis functions,$(j=1, \ldots, K)$, and

$$
u_{i-1}(x)=\sum_{j} U_{i-1}^{j} \Phi_{j}(x)
$$

Here, $U_{i-1}^{j}$ represents the value of the function $u_{i-1}(x)$ at the nodal point $x_{j}$. The errors in the evaluation of this inner product is a source of numerical instabilities. To evaluate the integrals (48) we follow the concept of 
Bermejo [6] and [7] (initiated by Morton et al., see [23]). That concept of evaluation, based on back-tracing, is unconditionally stable - see $[6,7]$.

We sketch the basic principles of the numerical implementation. The idea has emerged from noticing that the integral involving the product of two bilinear polynomials (in 2D) in different grid is equivalent to cubic spline interpolation at the knots of the displaced grid along the characteristic curves (when using $C^{\circ}$ finite elements with affine linear basis functions). Let $\varphi_{i}^{j}=\varphi_{i}\left(x_{j}\right), \varphi_{i}^{j}=\left(\varphi_{i}^{1, p}, \varphi_{i}^{2, q}\right)$, represents the displacement of the point $x_{j}$ along the characteristics, where $p(1 \leq L$ and $q(1 \leq P$ denote the index of the center point in the displaced element such that $j=(p-1) L+q$ and $1 \leq j \leq K(\equiv P . L)$. The integral (48) is denoted by $R$. Then, as shown by Bermejo in [7], $R$ is the value of a bicubic spline at the point $\varphi_{i}^{j}$, which is of the form

$$
R\left(\varphi_{i}^{1, p}, \varphi_{i}^{2, q}\right)=\sum_{s=1}^{L} \sum_{r=1}^{P} u_{i-1}^{r, 1} K_{r}\left(\varphi_{i}^{1, p}\right) K_{s}\left(\varphi_{i}^{2, q}\right) \equiv\left(u_{i-1} \circ \varphi_{i}, \Phi_{j}\right), \quad j=1, \ldots, K,
$$

where $p$ and $q$ determine $j$ and $K_{r}\left(\varphi_{i}^{1, p}\right)$ and $K_{s}\left(\varphi_{i}^{2, q}\right)$ are the values of the piecewise cubic polynomials in $\varphi_{i}^{1, p}$ and $\varphi_{i}^{2, q}$, respectively, and where $u_{i-1}$ is the solution at time level $t=t_{i-1}$. If we move $\varphi_{i}^{j}$ back to $x_{j}=\left(x_{1, p}, x_{2, q}\right)$ then

$$
R\left(x_{j}\right)=\left[A U_{i-1}\right]_{j}, \quad j=1, \ldots, K,
$$

where $A \equiv\left(a_{l, k}\right)$ is the matrix defined by

$$
a_{l, k}=\int_{\Omega} \Phi_{l} \Phi_{k} \mathrm{~d} x \quad \text { for } \quad l, k=1, \ldots, K
$$

and $\left[\begin{array}{lll}A & U_{i-1}\end{array}\right]_{j}$ denotes the $j-t h$ component of $\left[A U_{i-1}\right]$.

Now we can express $R\left(x_{j}\right)$ in terms of bicubic B-splines

$$
R(x)=\sum_{j} \nu_{i-1}^{j} B_{j}(x)
$$

where $B_{j}=B_{1, j} \otimes B_{2, j}$ is the Cartesian product of the natural cubic splines $B_{1, j}$ and $B_{2, j}$ corresponding to $x_{1}$ and $x_{2}$, respectively. The coefficients $\nu_{i-1}^{j}$ can be determined from the algebraic system (50). Then, we obtain

$$
\left(u_{i-1} \circ \varphi_{i}, \Phi_{j}\right)=R\left(\varphi_{i}^{1, p}, \varphi_{i}^{2, q}\right) \quad \text { for } \quad j=1, \ldots, P,
$$

where $j \leftrightarrow(p, q)$.

The degeneracy of the parabolic term is controlled by the relaxation scheme leading to the iterations (13-12).

Remark 7.1. In our numerical experiments we consider the non-equilibrium adsorption term in the form (6), where $\Lambda \equiv\{\emptyset, E\}$ with $\mu(\emptyset)=0, \mu(E)=1$. We assume that

$$
\varphi(\lambda, u) \equiv \varphi(u)=c u^{q}, \quad 0<q<1 .
$$

In that case we can integrate the ODE (2) and obtain

$$
v(t)=v_{0} \exp (-a t)+\int_{0}^{t} \exp (-a(t-s)) \varphi(u(s)) \mathrm{d} s, \quad a \equiv k(x) .
$$

Then, we substitute $\partial_{t} v$ in (1) by the expression

$$
s(t):=-v_{0} a \exp (-a t)+\varphi(u(t))-a \int_{0}^{t} \exp (-a(t-s)) \varphi(u(s)) \mathrm{d} s\left(=\partial_{t} v\right) .
$$


This expression is approximated in the following way - see [19]. In (7) we omit the non-equilibrium term (integral over $\lambda$ ). Moreover, in the R.H.S., $G_{i}$ is replaced by

$$
G_{i}^{\prime}=G_{i}+v_{0} a \exp \left(-a t_{i}\right)+\varphi\left(u_{i-1}\right)+s_{i}
$$

where

$$
s_{i}=a \sum_{j=1}^{i-1} \alpha_{i j} \varphi\left(u_{i}\right) \tau \quad \text { with } \quad \alpha_{i j}:=\frac{1}{\tau} \int_{t_{j-1}}^{t_{j}} \exp (-a(t-z)) \mathrm{d} z .
$$

This corresponds to the approximation of $u(t)$ by the piecewise constant function $\bar{u}^{n}(t)$. We can also approximate the memory term by using a piecewise linear approximation, when replacing $\bar{u}^{n}(t)$ by $u^{n}(t)$.

In our special case of non-equilibrium adsorption we can use the recurrence relation - see [19]

$$
s_{i+1}=s_{i} \exp (-a \tau)+\alpha_{i+1, i} \varphi\left(u_{i}\right) \quad \text { for } \quad i=1, \ldots, n
$$

This substantially simplifies the evaluation of the memory term, since we don't need to storage $u_{0}, \ldots, u_{i-1}$ for the evaluation of $s_{i}$.

\section{Numerical experiments in 1D}

We present only 1D numerical experiments supporting our concept of approximation; however this concept can also be used in the more dimensional case. We consider

$$
\begin{gathered}
\partial_{t} u+\varrho_{1} \partial_{t} u^{p}+\varrho_{2} \partial_{t} v+w \nabla u-D \Delta u=0 \quad \text { in } x \in(0, L), t>0, \\
\partial_{t} v=\kappa\left(u^{q}-v\right),
\end{gathered}
$$

with constant $\varrho_{1}, \varrho_{2}, q, D=0.05, w=6$, along with the Dirichlet boundary conditions

$$
u(0, t)=u(L, t)=0
$$

The initial profile $u_{0}(x)$ is given in pulse form, piecewise linear, with $u_{0}(0)=0, u_{0}(0.2)=u_{0}(0.4)=1$ and $u_{0}(x)=0$ for $x \geq 0.5$. We consider $v(x, 0) \equiv 0$. In the following examples $1-4$ we use as discretization parameters: $\Delta x=0.01, \Delta t=0.005, k=3$.

For simplicity, we eliminate the kinetic equation in (51) as described in Remark 7.1 and the first governing equation of (51) is considered with the corresponding memory term.

The concentration profile $u(x)$ at $\mathrm{t}=1,2$ and 6 is depicted in Figures 1-3 below for the following choices of $\varrho_{1}, \varrho_{2}$ and parameters $p, q, \kappa$ :

example 1: $\varrho_{1} \equiv 1, \varrho_{2} \equiv 0$; with the parameter $p=0.6$ : dotted line, $p=1$ : full line and $p=1.2$ : dashed line.

example 2: $\varrho_{1} \equiv 0, \varrho_{2} \equiv 1$; with the parameter $\kappa=5$ and with the parameter $q=0.6$ : dotted line, $q=1$ : full line and $q=1.2$ : dashed line.

example 3: $\varrho_{1} \equiv \varrho_{2} \equiv 1$; with the parameters $\kappa=5, q=0.6$ and with the parameter $p=0.6$ : dotted line, $p=1$ : full line and $p=1.2$ : dashed line.

example 4: $\varrho_{1} \equiv \varrho_{2} \equiv 0$.

In the last example the concentration and speed of propagation is higher then that in the cases with adsorption.

\section{Construction of the analytic solution to a model problem}

To demonstrate the effectiveness of our numerical scheme, we will compare the numerical solution with the analytical one for a problem where the latter can be constructed in an heuristic way. We shall consider the 

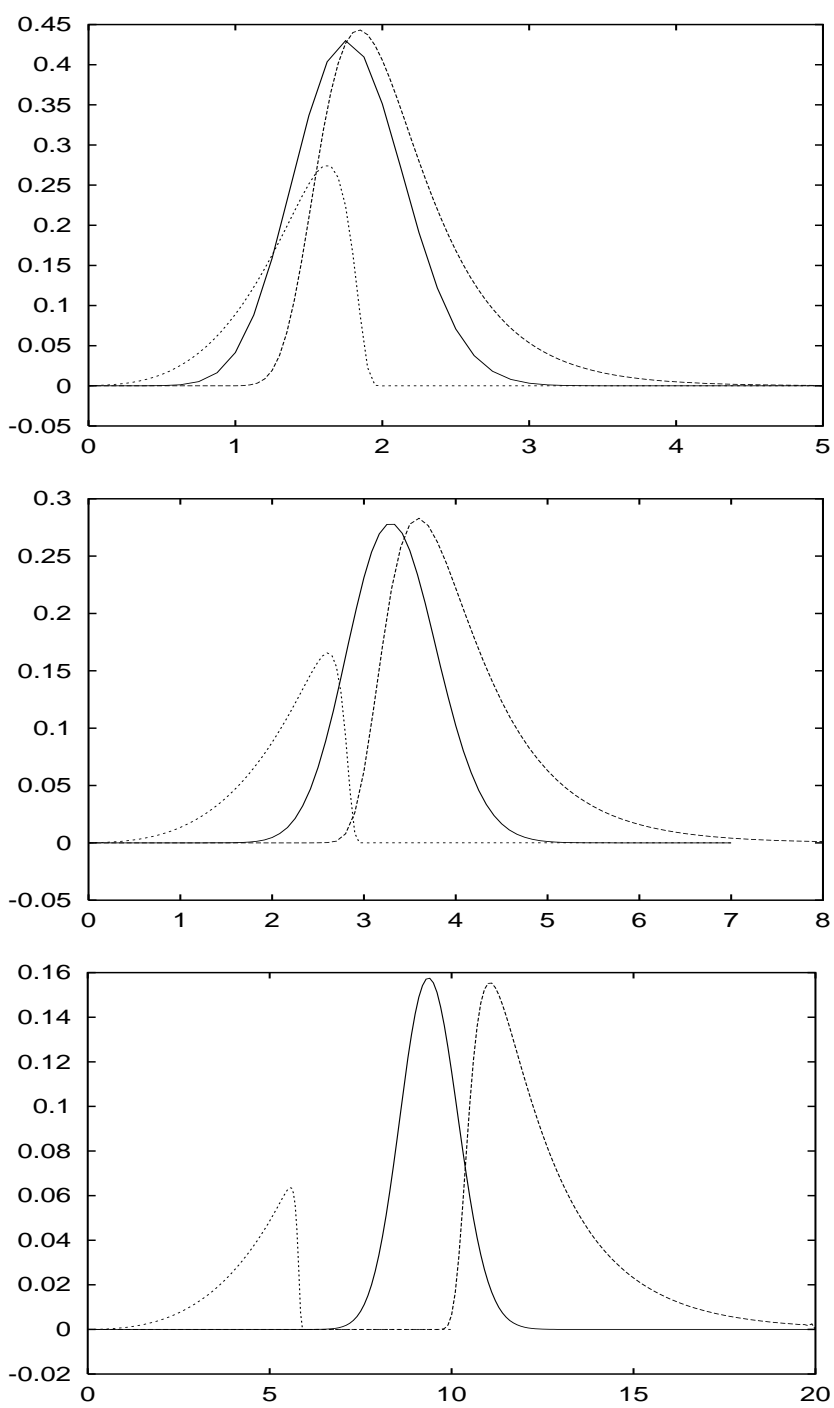

Figure 1. Example 1: concentration profiles at $t=1,2$ and 6.

following model problem:

$$
\begin{gathered}
\partial_{t}\left(u+u^{1 / 2}\right)+\partial_{x} u-D \partial_{x}^{2} u=0, \quad x>0, t>0, \\
u(0, t)=u_{0} \psi(t), \quad u(x, 0)=0, x>0
\end{gathered}
$$

where $\psi$ is a smooth increasing function with $\psi(0)=0$ and $\psi(t) \rightarrow 1$ for $t \rightarrow \infty$. We shall specify it later. The existence of a unique solution is guaranteed in [21,25].

Notice that (52) is a special case of (51), where adsorption in equilibrium mode is considered only.

We can expect the existence of a traveling wave type solution, since the adsorption (represented by $u^{1 / 2}$ ) is strong and implies the solution to have a finite support.

We are looking for the solution of the form $u(x, t)=f(x-v t)$, where we have to find the constant $v$ and the wave profile $f=f(\xi)$. We expect $f(\xi)$ to be decreasing from $u_{0}$ to 0 , with $f(\xi) \rightarrow u_{0}$ for $\xi \rightarrow-\infty$ and $f(\xi)=0$ for $\xi \in(L, \infty)$ (finite support). Then $\psi(t)=\frac{1}{u_{0}} f(-v t)$. 

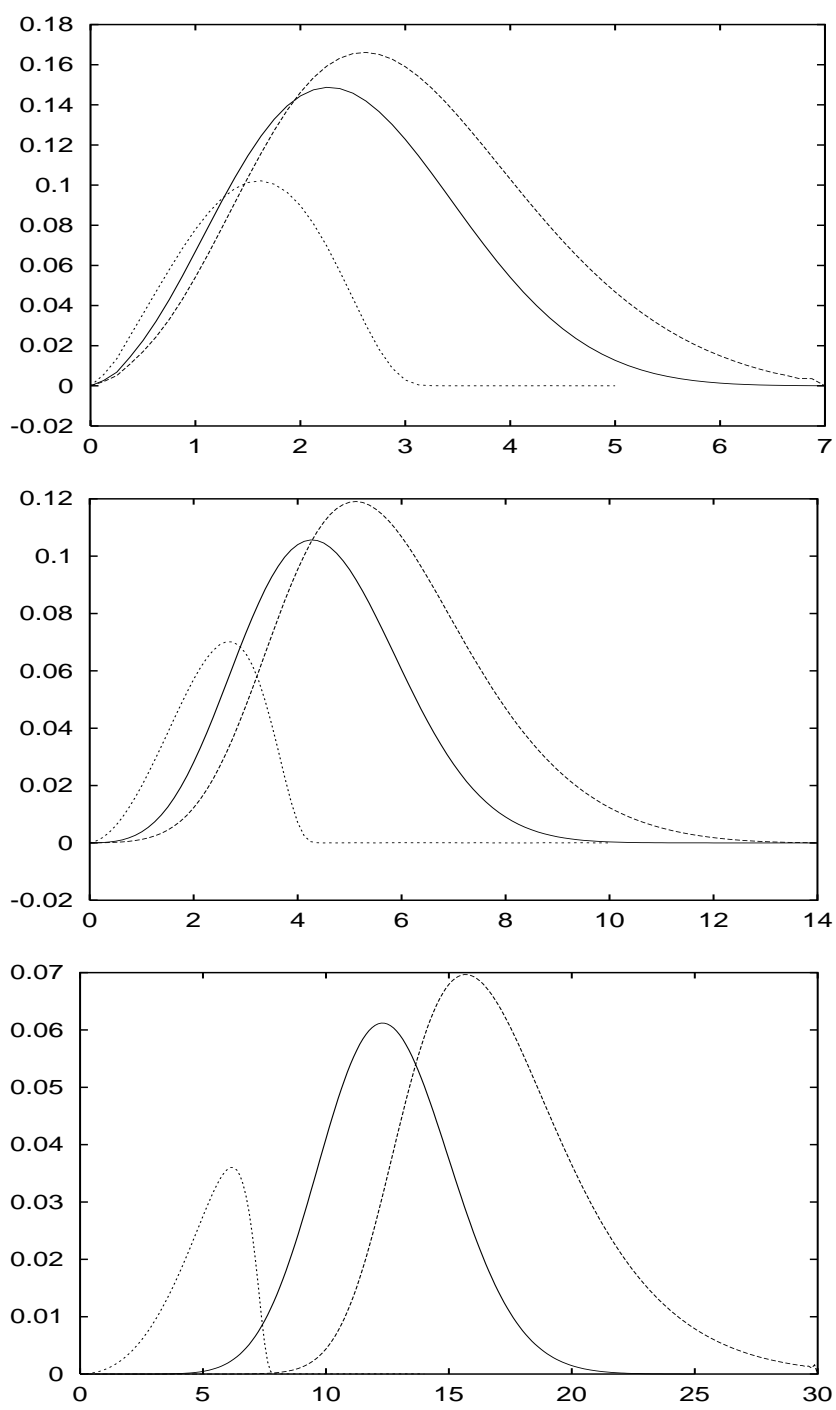

Figure 2. Example 2: concentration profiles at $t=1,2$ and 6.

For $f$ we obtain the following ODE

$$
f^{\prime}\left[1-v-\frac{1}{2} v f^{-1 / 2}\right]=D f^{\prime \prime}
$$

We are looking for $W=W(f)$ satisfying $W(f)=f^{\prime}$. The governing ODE for $W$ is

$$
W^{\prime}=\frac{1}{D}\left[1-v-\frac{1}{2} v f^{-1 / 2}\right] .
$$

We can put $f=z^{2}, z>0$, and $u(z)=W(f)$. Then,

$$
u^{\prime}=\frac{2}{D}\left[(1-v) z-\frac{1}{2} v\right] .
$$



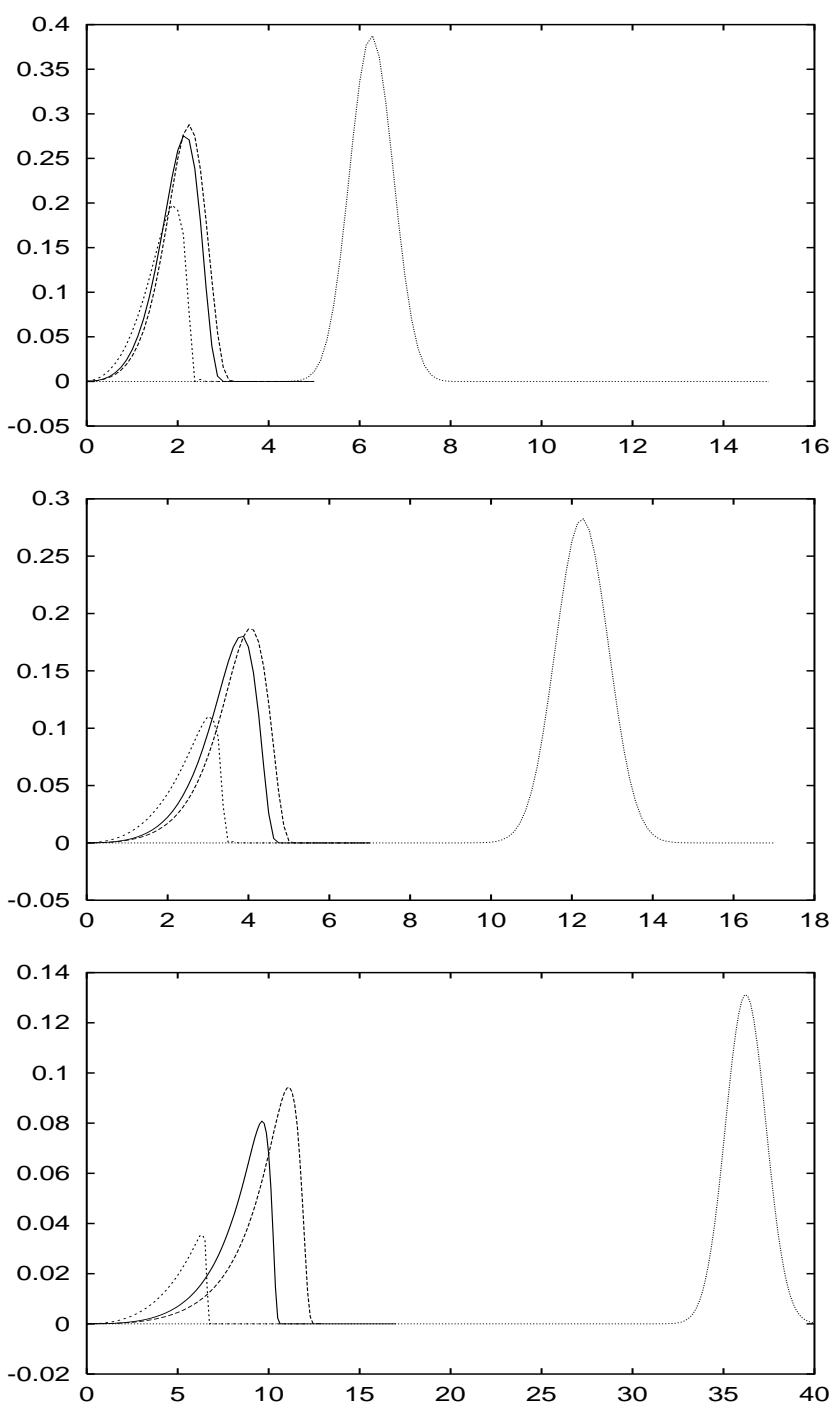

Figure 3. Example 3 and 4: concentration profiles at $t=1,2$ and 6 .

and, consequently,

$$
u(z)=\frac{2}{D}\left[\frac{1-v}{2} z^{2}-\frac{1}{2} v z\right]+C_{1} .
$$

We take $C_{1}=0$. From the definitions of $u$ and $W$ in terms of $f$, it follows that

$$
\int \frac{\mathrm{d} f}{\frac{1-v}{2} f-\frac{1}{2} v f^{1 / 2}}=\frac{2}{D} \int \mathrm{d} \xi
$$

and hence

$$
\ln \left|\frac{1-v}{2} f^{1 / 2}-\frac{1}{2} v\right|=\frac{1}{2 D}(1-v) \xi+\tilde{C}_{2} .
$$


Consequently,

$$
f(\xi)=\left(\frac{v}{1-v}+C_{2} \exp \left(\frac{1}{2 D}(1-v) \xi\right)\right)^{2}
$$

To guarantee that $f(\xi) \rightarrow u_{0}$ for $\xi \rightarrow-\infty$, we have to put $v=\frac{\sqrt{u_{0}}}{1+\sqrt{u_{0}}}$. To guarantee that $f(\xi)$ has a positive root (in view of the construction of a solution with finite support), we take $C_{2}<0$. More specifically, we require that $\psi(0)=f(0)=0$, which implies $C_{2}=-\frac{v}{1-v}$.

From these considerations we conclude that

$$
u(x, t)=\left\{\begin{array}{l}
u_{0}\left(1-\exp \left(\frac{1}{2 D} \frac{1}{1+\sqrt{u_{0}}} \xi\right)\right)^{2}, \quad \xi<0, \\
0, \quad \xi>0
\end{array}\right.
$$

with

$$
\xi=x-v t, \quad v=\frac{\sqrt{u_{0}}}{1+\sqrt{u_{0}}},
$$

constitutes a formal traveling wave solution to the BVP (52), where we have taken $\psi(t)=\frac{1}{u_{0}} f(-v t)$. This formal solution is readily verified.

In our numerical experiments we take $u_{0}=1$ and hence $v=\frac{1}{2}$ and $\psi(t)=\left(1-\exp \left(-\frac{t}{8 D}\right)\right)^{2}$. Since D is small, $\psi(t)$ is very close to 1 for $t>\delta>0$ with small $\delta$.

\section{Comparison of numerical with exact solutions}

For the model problem above we use approximation Scheme $\mathrm{I}_{k}$, which coincides with $\mathrm{Scheme} \mathrm{II}_{k}$, due to the absence of an adsorption term in the non-equilibrium mode. The considered adsorption in the equilibrium mode causes the presence of sharp fronts in the solution. This is captured by means of the relaxation iterations (in $k$ ) and by the method of characteristics included in Schemes $\mathrm{I}_{k}$ and $\mathrm{II}_{k}$. Our type of approximation can be seen to be efficient in the following experiments, graphically presented in Figure 4 and Figure 5.

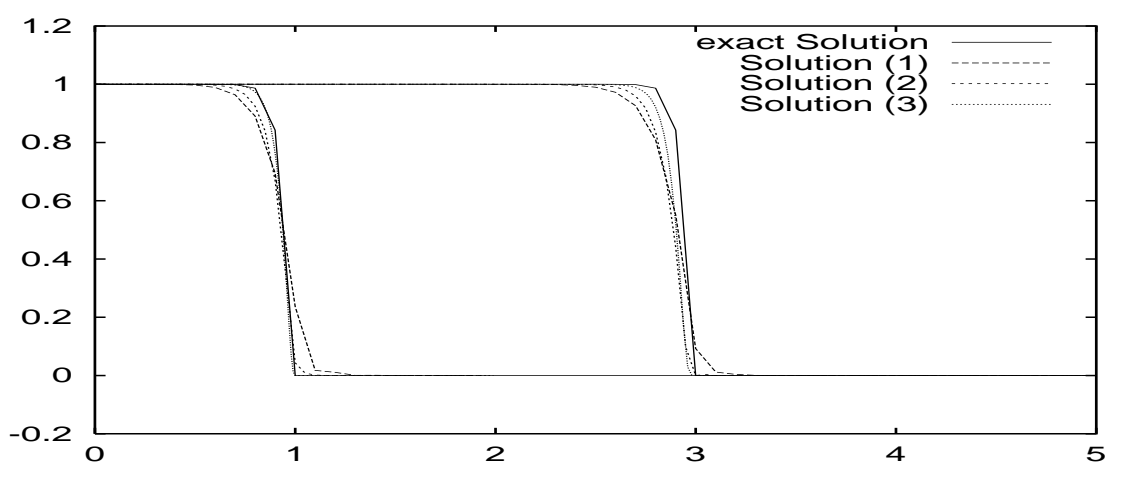

Figure 4. Comparison with exact solution for example 5 at $t=2$ and $t=6$.

example 5: We use the data $u_{0}=1.0, D=0.01$, with the discretization and iteration parameters listed in Table 1. In Figure 4 we present the exact and numerical solutions with the corresponding discretization parameters for $t=2$ and $t=6$. In this case the Courant number equals 1.

example 6: We consider a more regular case with stronger diffusion. We take $u_{0}=1, D=0.1$, with the discretization and iteration parameters in Table 2. Less iteration steps have been used due to the higher regularity of the solution. The comparison with the exact solution is depicted in Figure 5 for $t=2$ and $t=6$. The real velocity (retarded) of contaminant transport equals $\frac{1}{2}$, while the water velocity $w=1$. 


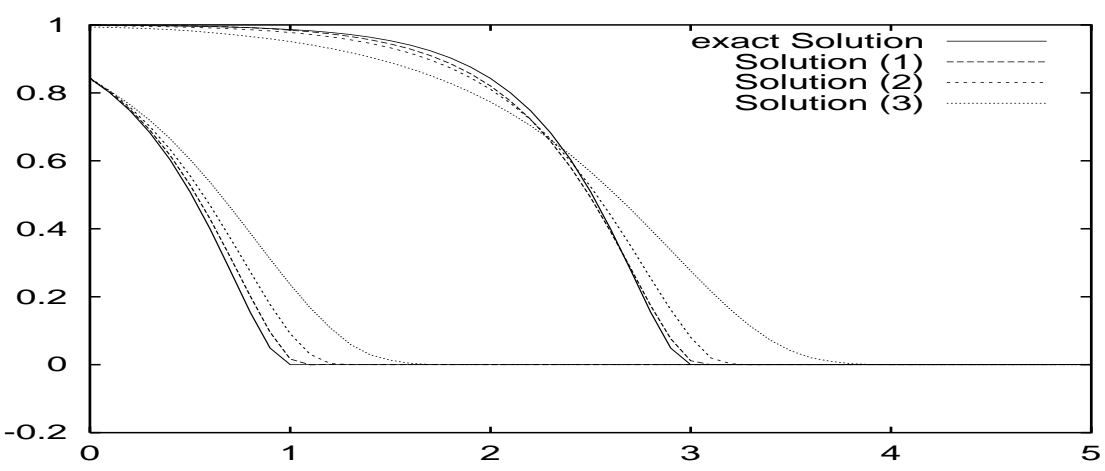

Figure 5. Comparison with exact solution for example 6 at $t=2$ and $t=6$.

TABLE 1. Discretization and iteration parameters for example 5.

\begin{tabular}{ccccc}
\hline Solution & Number of iterations $(\mathrm{k})$ & Time & Time step & Space step \\
\hline 1 & 3 & $t=2$ & $\Delta t=1 / 10$ & $\Delta x=1 / 10$ \\
\hline 2 & 3 & $t=2$ & $\Delta t=1 / 20$ & $\Delta x=1 / 20$ \\
\hline 3 & 3 & $t=2$ & $\Delta t=1 / 50$ & $\Delta x=1 / 50$ \\
\hline 1 & 5 & $t=6$ & $\Delta t=1 / 10$ & $\Delta x=1 / 10$ \\
\hline 2 & 5 & $t=6$ & $\Delta t=1 / 20$ & $\Delta x=1 / 20$ \\
\hline 3 & 5 & $t=6$ & $\Delta t=1 / 50$ & $\Delta x=1 / 50$ \\
\hline
\end{tabular}

TABLE 2. Discretization and iteration parameters for example 6.

\begin{tabular}{cclll}
\hline Solution & Number of iterations k & Time & Time step & Space step \\
\hline 1 & 3 & $t=2$ & $\Delta t=1 / 10$ & $\Delta x=1 / 10$ \\
\hline 2 & 3 & $t=2$ & $\Delta t=1 / 5$ & $\Delta x=1 / 10$ \\
\hline 3 & 3 & $t=2$ & $\Delta t=1 / 2$ & $\Delta x=1 / 10$ \\
\hline 1 & 3 & $t=6$ & $\Delta t=1 / 10$ & $\Delta x=1 / 10$ \\
\hline 2 & 3 & $t=6$ & $\Delta t=1 / 5$ & $\Delta x=1 / 10$ \\
\hline 3 & 3 & $t=6$ & $\Delta t=1 / 2$ & $\Delta x=1 / 10$ \\
\hline
\end{tabular}

The approximate solutions 2 and 3 correspond to the discretization parameters with the Courant number larger then 1.

Remark 7.2. The convergence of the relaxation scheme with respect to the iteration parameter $k$ is analyzed in [17], where an equilibrium type adsorption term has been considered only. A similar situation occurs when also a non-equilibrium term is present - see Scheme $\mathrm{II}_{k}$. A Newton type approximation of the equilibrium type adsorption term requires a smaller time step then in our relaxation concept. For large time steps the Newton type approximation can fail. For a sufficiently small time step the Newton type approximation converges more quickly. In our numerical experiments it was sufficient to take $k$ between 3 and 7 . Due to the used method of characteristics we observe numerical stability also for discretization parameters with Courant number larger then 1. In our numerical experiments we have approximated the non-equilibrium type adsorption term as explained in Remark 7.1. In a similar way as in Schemes $\mathrm{I}_{k}$ and $\mathrm{II}_{k}$ also more general adsorption terms e.g. of competitive type can be approximated. In that case in each time step (after transport and diffusion) we have to solve some algebraic nonlinear system. The convergence analysis in this case is an open question. 
Acknowledgements. The authors express their thanks to Dr. Mohammed Shuker for his help with the numerical experiments. The authors also gratefully acknowledge the financial support from the project BOF/GOA 12051598 of Ghent University. The first author was also partially supported by GACR 201/00/0557.

\section{REFERENCES}

[1] H.W. Alt and S. Luckhaus, Quasilinear elliptic-parabolic differential equations. Math. Z. 183 (1983) 311-341.

[2] J.W. Barrett and P. Knabner, Finite element approximation of transport of reactive solutes in porous media. Part i: error estimates for nonequilibrium adsorption processes. SIAM J. Numer. Anal. 34 (1997) 201-227.

[3] J.W. Barrett and P. Knabner, Finite element approximation of transport of reactive solutes in porous media. Part ii: error estimates for equilibrium adsorption processes. SIAM J. Numer. Anal. 34 (1997) 455-479.

[4] J.W Barrett and P. Knabner, An improved error bound for a Lagrange-Galerkin method for contaminant transport with non-Lipschitzian adsorption kinetics. SIAM J. Numer. Anal. 35 (1998) 1862-1882.

[5] J. Bear, Dynamics of Fluids in Porous Media. Elsevier, New York (1972).

[6] R. Bermejo, Analysis of an algorithm for the Galerkin-characteristics method. Numer. Math. 60 (1991) 163-194.

[7] R. Bermejo, A Galerkin-characteristics algorithm for transport-diffusion equation. SIAM J. Numer. Anal. 32 (1995) $425-455$.

[8] C.N. Dawson, Godunov-mixed methods for advection diffusion equations in multidimensions. SIAM J. Numer. Anal. 30 (1993) $1315-1332$.

[9] C.N. Dawson, Analysis of an upwind-mixed finite element method for nonlinear contaminant transport equations. SIAM J. Numer. Anal. 35 (1998) 1709-1724.

[10] C.N. Dawson, C.J. van Duijn, and R.E. Grundy, Large time asymptotics in contaminant transport in porous media. SIAM J. Appl. Math. 56 (1996) 965-993.

[11] C.N. Dawson, C.J. van Duijn, and M.F. Wheeler, Characteristic-Galerkin methods for contaminant transport with nonequilibrium adsorption kinetics. SIAM J. Numer. Anal. 31 (1994) 982-999.

[12] R Douglas and T.F. Russel, Numerical methods for convection dominated diffusion problems based on combining the method of characteristics with finite element or finite difference procedures. SIAM J. Numer. Anal. 19 (1982) 871-885.

[13] N. Dunford and J.T. Schwartz, Linear Operators. Part I: General Theory. John Wiley \& Sons Ltd., New York (1959).

[14] R.E. Grundy, C.J. van Duijn, and C.N. Dawson, Asymptotic profiles with finite mass in one-dimensional contaminant transport through porous media. Quart. J. Mech. Appl. Math. 1 (1994) 69-106.

[15] W. Jäger and J. Kačur, Solution of doubly nonlinear and degenerate parabolic problems by relaxation schemes. RAIRO Modél. Math. Anal. Numér. 29 (1995) 605-627.

[16] J. Kačur, Solution of some free boundary problems by relaxation schemes. SIAM J. Numer. Anal. 36 (1999) $290-316$.

[17] J. Kačur, Solution to strongly nonlinear parabolic problems by a linear approximation scheme. IMA J. Numer. Anal. 19 (1999) $119-154$.

[18] J. Kačur and S. Luckhaus, Approximation of degenerate parabolic systems by nondegenerate elliptic and parabolic systems. Appl. Numer. Math. 25 (1997) 1-21.

[19] J. Kačur, Solution of convection-diffusion problems with the memory terms, in Applied Mathematical Analysis, A. Sequiera, H. Beirao de Veiga, and J.H. Videman, Eds., Kluwer Academic, Plenum Publ., New York (1999) 199-212.

[20] P. Knabner, Mathematische Modelle für den Transport gelöstes Stoffe in sorbierenden Porösen Medien. Habilitationschrift, University of Augsburg, Germany (1989).

[21] P. Knabner and F. Otto, Solute transport in porous media with equilibrium and nonequilibrium multiple site adsorption: uniqueness. To appear.

[22] A. Kufner, O. John, and S. Fučík, Function Spaces. Noordhoff International Publishing, Leyden; Publishing House of the Czechoslovak Academy of Sciences, Prague (1977).

[23] K.W. Morton, A. Priestly, and E. Suli, Stability of the Lagrange-Galerkin method with non-exact integration. RAIRO Modél. Math. Anal. Numér. 4 (1988) 225-250.

[24] J. Nečas, Les méthodes directes en théorie des équations elliptiques. Academia, Prague (1967).

[25] F. Otto, L1-contraction and uniqueness for quasilinear elliptic-parabolic equations. C. R. Acad. Sci. Paris Sér. I Math. 321 (1995) 105-110.

[26] P. Pironneau, On the transport-diffusion algorithm and its application to the Navier-Stokes equations. Numer. Math. 38 (1982) 309-332.

[27] C.J. van Duijn and P. Knabner, Solute transport in porous media with equilibrium and nonequilibrium multiple site adsorption: Traveling waves. J. Reine Angew. Math. 415 (1991) 1-49.

To access this journal online:

www.edpsciences.org 